\title{
Metabolomic Profiling, Antioxidant and Antimicrobial Activity of Bidens pilosa
}

\author{
Paola Angelini ${ }^{1,+}{ }^{\oplus}$, Florentina Matei ${ }^{2,+}$, Giancarlo Angeles Flores ${ }^{1}$, Roberto Maria Pellegrino ${ }^{1}(\mathbb{0}$, \\ Lydie Vuguziga ${ }^{2, *}$, Roberto Venanzoni ${ }^{1}{ }^{10}$, Bruno Tirillini ${ }^{3}$, Carla Emiliani ${ }^{1}$, Giustino Orlando ${ }^{4}$, \\ Luigi Menghini ${ }^{4, *}$ and Claudio Ferrante ${ }^{4}$ (D)
}

1 Department of Chemistry, Biology and Biotechnology, University of Perugia, 06100 Perugia, Italy; paola.angelini@unipg.it (P.A.); giancarlo.angelesflores@studenti.unipg.it (G.A.F.); roberto.pellegrino@unipg.it (R.M.P.); roberto.venanzoni@unipg.it (R.V.); carla.emiliani@unipg.it (C.E.)

2 Faculty of Biotechnologies, University of Agronomic Sciences and Veterinary Medicine of Bucharest, 011464 Bucharest, Romania; florentina.matei@biotehnologii.usamv.ro

3 Department of Biomolecular Sciences, University of Urbino, 61029 Urbino, Italy; bruno.tirillini@uniurb.it

4 Department of Pharmacy, Università degli Studi "Gabriele d'Annunzio", via dei Vestini 31, 66100 Chieti, Italy; giustino.orlando@unich.it (G.O.); claudio.ferrante@unich.it (C.F.)

* Correspondence: lydika1@yahoo.fr (L.V.); luigi.menghini@unich.it (L.M.)

+ Both authors contributed equally to this work.

\section{check for} updates

Citation: Angelini, P.; Matei, F.; Flores, G.A.; Pellegrino, R.M.;

Vuguziga, L.; Venanzoni, R.; Tirillini,

B.; Emiliani, C.; Orlando, G.;

Menghini, L.; et al. Metabolomic

Profiling, Antioxidant and

Antimicrobial Activity of Bidens pilosa.

Processes 2021, 9, 903. https://

doi.org/10.3390/pr9060903

Academic Editors: Adriana Trifan, Luigi Menghini and Claudio Ferrante

Received: 27 April 2021

Accepted: 17 May 2021

Published: 21 May 2021

Publisher's Note: MDPI stays neutral with regard to jurisdictional claims in published maps and institutional affiliations.

Copyright: (c) 2021 by the authors. Licensee MDPI, Basel, Switzerland. This article is an open access article distributed under the terms and conditions of the Creative Commons Attribution (CC BY) license (https:/ / creativecommons.org/licenses/by/ $4.0 /)$.
Abstract: Bidens pilosa L. (fam. Asteraceae) is an annual herb used globally in phytotherapy and each plant material or the whole plant have been declared to be effective. Therefore, the aim of the present study was to conduct metabolomic profiling of different plant materials, including the quali-quantitative composition of phenolic compounds. The intrinsic scavenging/reducing properties and antimicrobial effects of the extracts were assayed against numerous bacterial, Candida and dermatophytes species, whereas docking runs were conducted for tentatively unravelling the mechanism of action underlying antimicrobial effects. Oligosaccharide, disaccharide and fatty acids were present at higher concentrations in root rather than in the other plant parts. Monoglycerides were more abundant in stem than in the other plant parts, whereas peptide and diterpenoid were prominent in leaf and root, respectively. By contrast, amino acids showed very different distribution patterns in the four plant parts. Regarding the phenolic composition, appreciable levels of caftaric acid were found in most of the analyzed methanol extracts, that were also particularly efficacious as antiradical and anti-mycotic agents against $C$. albicans and dermatophytes. The docking experiments also showed a micromolar affinity of caftaric acid towards the lanosterol $14 \alpha$-demethylase, deeply involved in fungal metabolism. In conclusion, the present study corroborates the B. pilosa as a phytotherapy remedy against infectious disease.

Keywords: Bidens pilosa; metabolomic profile; anti-mycotic effects; phenolic compounds; caftaric acid; bioinformatics

\section{Introduction}

Plants are an important source of pharmacologically active secondary compounds, that can be used in medicine to maintain and improve human health and also to treat specific conditions or illnesses [1,2]. Among these compounds, phenolics are very common and easy to find in plants, and they have demonstrated beneficial advantages in terms of antioxidant and antimicrobial activities. In particular, antioxidant metabolites can be used against different oxidative stress-induced diseases [3], while the antimicrobial properties of such compounds can rehabilitate the clinical application of older antibiotics by improving their efficacy and, therefore, by preventing the development of resistance [4]. Bidens pilosa L. (fam. Asteraceae) is an annual herb native to South America that is spread worldwide, especially in tropical and subtropical regions [5]. B. pilosa L. is used globally in phytotherapy 
and each plant material or the whole plant have been declared to be effective in treating many illnesses such as malaria, flu, cancers, headache, inflammation, wounds, angina, metabolic syndrome, immunological disorders, and digestive and infectious diseases [6]. The plant has been widely used in Taiwan as a traditional medicine and as a major ingredient of herbal tea, which is believed to prevent inflammation and cancer [7]. Phytochemical and pharmacological analyses of B. pilosa employing roots [8], leaves [9], or the whole aerial parts $[10,11]$ have also been published. In this regard, the studies indicated the presence of phenolic compounds that could explain, albeit partially, antioxidant and antimicrobial activities [12-20]. In a study by Abajo et al. (2004) [15], the antioxidant activity of an aqueous infusion of $B$. pilosa has been investigated by studying its protective effect on the hemolysis induced by an initiator of radicals such as 2,2'-azobis(2-amidinopropane) dihydrochloride (AAPH). The amount of $B$. pilosa infusion that halved the hemolysis induced by AAPH was $6 \mu \mathrm{L}$ (IC50: $1.19 \mathrm{mg} \mathrm{mL}^{-1}$ dry weight). Chiang and colleagues (2004) [21] evaluated the free radical scavenging activity of the crude extract, and fractions of $B$. pilosa using 1,1-diphenyl-2-picrylhydrazyl (DPPH) and hypoxanthine/xanthine oxidase assays. They found that the $B$. pilosa crude extract and the ethyl acetate, butanol, and water fractions had free radical scavenging activity and that the ethyl acetate and butanol fractions were more active than the water fraction and crude extract [21]. A complementary study by Muchuweti et al. (2007) [22] determined antioxidant activity of B. pilosa methanol extract. It also showed 1,1-diphenyl-2-picrylhydrazyl (DPPH) radical scavenging activity [22]. Ashafa et al. (2009) [18] reported that the methanol and acetone extract of B. pilosa roots displayed antibacterial activities against Bacillus cereus, Escherichia coli, Klebsilla pneumonia, Micrococcus kristinae, Pseudomonas aeruginosa, Staphylococcus aureus, S. epidermidis, Serratia marcescens, Shigelea flexneri, and Streptococcus faecalis. Deba et al. (2007) [23] first evaluated the antifungal effect of the hot water extracts of the B. pilosa roots, stems, and leaves against Corticium rolfsii, Fusarium solani, and Fusarium oxysporum. C. rolfsii was particularly sensitive to the treatment with $B$. pilosa as its growth was reduced at almost all the tested concentrations, followed by F. oxysporum and F. solani. However, the fungicidal activities of the stems and roots were greater than the leaves [23]. The composition analysis of the extracts revealed the presence of different phenolic compounds that could be at the basis of the fungicidal effects. Essential oils appeared to have better fungicidal activity than water extracts [17]. Acetone, methanol, and water extracts of the B. pilosa roots also showed antifungal activities against Aspergillus niger, A. flavus, and Penicillium notatum using the agar dilution method [18]. The methanol extract of the B. pilosa roots at $10 \mathrm{mg} / \mathrm{mL}$ was also effective against Candida albicans [18]. Shandukani et al. (2018) [20] investigated the B. pilosa antibacterial activity against waterborne diarrhoeagenic bacteria. All the bacterial species tested were sensitive to the effect of different extracts of B. pilosa. Moreover, Nthulane et al. (2020) [24] determined the antimicrobial activities of plant extracts against the bacteria causing common sexually transmitted infections. The results showed that dichloromethane extract of B. pilosa exhibited good activities against Neisseria gonorrhoeae and Gardnerella vaginalis, whereas ethyl acetate, dichloromethane and methanol extracts of $B$. pilosa exhibited good activities against C. albicans. Some classes of compounds such as flavonoids, aliphatics, terpenoids, phenylpropanoids, aromatics and porphyrins were isolated from B. pilosa and related to the bio-pharmacological properties of this plant $[5,19]$. Also, saponins and steroids were identified in the phytocomplex of $B$. pilosa. These compounds were suggested to be involved in the antioxidant [21], antibacterial and antimicrobial activities [5,20]. In recent years, metabolomics, which is defined as the monitoring of metabolite concentration in a cell, tissue, organ or in the whole plant, has become prominent as a part of systems biology. Nonetheless, differentiation between different plant materials of B. pilosa based on their metabolomic profiling has not been carried out yet. For a suitable comparative antioxidant and antimicrobial activity of different plant materials of this precious plant, we proposed the in vitro antimicrobial activity study of the roots, leaves, stems and the whole plant extracts of $B$. pilosa by using various solvents. This study evaluates such activity towards some selected Gram-positive, Gram-negative bacteria and fungal species. Further- 
more, a mass spectrometry ultra-performance liquid chromatography mass spectrometry (UHPLC)-QTOF method, coupled with different multivariate data analyses such as principle component analysis (PCA), was applied to B. pilosa metabolome aiming at investigating the metabolomic variation among the different plant materials of the same species and at evaluating this species as a potential antioxidant and antimicrobial. A liquid chromatography coupled to diode array and mass spectrometer (HPLC-DAD-MS) analysis was also conducted for measuring the levels of phenolic compounds in the extracts, whereas the intrinsic scavenging/reducing properties were determined via colorimetric methods. Finally, a docking approach was carried out for unravelling the putative mechanisms underlying the observed antimicrobial effects.

\section{Materials and Methods}

\subsection{Chemical and Reagents}

Mueller-Hinton broth (MHB), Rose Bengal Chloramphenicol Agar (RBCA), Malt Extract Agar (MEA), Sabouraud Dextrose Agar (SDA), RPMI (Roswell Park Memorial Institute) 1640 medium, and purity grade organic solvents (n-hexane, Ethyl acetate, Methanol, and Dimethyl Sulfoxide), were purchased from Sigma (Sigma-Aldrich, Milan, Italy).

\subsection{Plants Material}

The mature seeds of B. pilosa L. were collected in July 2018, at an altitude of 1500-1800 m in Yabaramba zone, Kicukiro district, Rwanda. The seeds were cleaned and sterilized with ethylic alcohol solution $70 \%$ for $1 \mathrm{~min}$ and washed thoroughly 3 times with sterile distilled water. The sterilised seeds were planted in a garden pot containing the sterilised garden soil with NPK 12:11:18:2. The plants full grown were separated into roots, leaves and stems. The plant materials were separated into 4 samples that are leaves, roots, stems and whole plants. Afterwards, they were dried in an autoclave at $40{ }^{\circ} \mathrm{C}$. The dried plant materials separated in leaves, roots, stems and whole plants were finely grounded and macerated in methanol for 7 days at $20^{\circ} \mathrm{C}(1: 10 w / v)$. The resulting extracts were then filtered through Whatman GF/C filters (Sigma, Germany), and the solvent evaporated under reduced pressure $\left(40^{\circ} \mathrm{C}, 218 \mathrm{mbar}\right)$ using a rotary vacuum evaporator (Rotavapor $\mathrm{R}-100$, Büchi, Switzerland). The residue was kept at $-20^{\circ} \mathrm{C}$ until further use.

\subsection{Untargeted LC-MS/MS-Based Metabolomics}

Untargeted metabolomics was carried out by using ultra-performance liquid chromatography mass spectrometry (UHPLC)-QTOF employing a 1260 ultra-high-performance liquid chromatograph and a G6530A QTOF mass spectrometer (Agilent Technologies, Santa Clara, CA, USA). An Agilen JetStream ionization source in positive and negative polarity was also used. LC separation was performed using an Ascentis Express Peptide ES-C18 Supelco column $(2.1 \times 750 \mathrm{~mm}, 2.7 \mu \mathrm{m})$ with a gradient elution of mobile phase A (water $+0.1 \%$ Formic Acid) and mobile phase B (Acetonitrile with $0.1 \%$ Formic Acid) LC gradient consisted of holding solvent (A/B: 98/2) for $2 \mathrm{~min}$, then linearly converting to solvent (A/B: 40/60) for $5 \mathrm{~min}$, linearly converting to solvent (A/B: 20/80) for $1 \mathrm{~min}$ and holding for $2 \mathrm{~min}$, then linearly converting to solvent (A/B: 98/2) for $0.5 \mathrm{~min}$, and holding for $3 \mathrm{~min}$ for re-equilibration. The flow rate and column temperature were set to $0.45 \mathrm{~mL} / \mathrm{min}$ and $45^{\circ} \mathrm{C}$, respectively. The mass spectrometer was operated in iterative Data Dependent Acquisition mode (50-1000 m/z), with a nominal resolution of 40,000 FWHM (full width at half maximum) and in the extended dynamic range mode using 5 precursors per cycle with collision energies of $30 \mathrm{eV}$. Peak picking and alignment were performed by MS-DIAL (ver. 4.38) with the following parameters: accurate mass tolerance (MS1) tolerance, $0.01 \mathrm{Da}$; MS2 tolerance, $0.025 \mathrm{Da}$; maximum charge number, two; smoothing method, linear weighted moving average; smoothing level, 3; minimum peak width, five scans; minimum peak height, 1000; mass slice width, $0.1 \mathrm{Da}$; sigma window value, 0.5; MS2Dec amplitude cut off, 0; exclude after precursor, true; keep isotope until, 0.5 Da; relative abundance cut off, 0 ; top candidate report, true; retention time tolerance for alignment, 
$0.1 \mathrm{~min}$; MS1 tolerance for alignment, $0.015 \mathrm{Da}$; peak count filter, 0; adduct ion setting, $[\mathrm{M}+\mathrm{H}]+,[\mathrm{M}+\mathrm{NH} 4]+,[\mathrm{M}+\mathrm{Na}]+$, in positive ion mode and $[\mathrm{M}-\mathrm{H}]-,[\mathrm{M}+\mathrm{CH} 3 \mathrm{COO}]-$ in negative ion mode. Compound annotation was made comparing the experimental MS/MS spectra to those available in the NIST2020 Tandem Mass Spectral Library. An $\mathrm{m} / \mathrm{z}$ window of $0.005 \mathrm{Da}$ and a relative intensity threshold of 0.5 were selected as input parameters. Only the compounds with an identification score cut off $>80 \%$ were retained for further analysis. Principle component analysis (PCA) and Heatmap were performed with MetoboAnalyst 5.0 for either annotated metabolites or ontology grouped metabolites. For PCA and Heatmap, samples were normalized by median, followed by pareto scaling.

\subsection{Determination of the Antioxidant Activity}

The antiradical activity was determined by the 1,1-diphenyl-2-picrylhydrazyl (DPPH) radical-scavenging method. Each sample was mixed with $900 \mu \mathrm{L}$ of $100 \mathrm{mM}$ Tris- $\mathrm{HCl}$ buffer, $\mathrm{pH} 7.4$, and then added to $1 \mathrm{~mL}$ of $0.5 \mathrm{mM}$ DPPH in methanol $(250 \mu \mathrm{M}$ in the reaction mixture). The control sample was prepared using methanol. Trolox was employed as a reference antioxidant substance. Absorbances of the mixtures were measured at $517 \mathrm{~nm}$. The activity was calculated as $\mathrm{IC}_{50}$ Trolox equivalent. All tests and analyses were run in triplicate and averaged. For ABTS (2,2'-azinobis-(3-ethylbenzothiazoline-6-sulphonate) radical scavenging) assay, the procedure followed the method of Arnao et al. with some modifications. The stock solutions included $7 \mathrm{mM}$ ABTS solution and $2.45 \mathrm{mM}$ potassium persulfate solution. The working solution was then prepared by mixing the two stock solutions in equal quantities and allowing them to react for $14 \mathrm{~h}$ at room temperature in the dark. The solution was then diluted by mixing $1 \mathrm{~mL}$ ABTS solution with $60 \mathrm{~mL}$ methanol to obtain an absorbance of $0.706 \pm 0.01$ units at $734 \mathrm{~nm}$. Fresh ABTS solution was prepared for each assay. Plant extracts $(1 \mathrm{~mL})$ were allowed to react with $1 \mathrm{~mL}$ of the ABTS solution and the absorbance was taken at $734 \mathrm{~nm}$ after $7 \mathrm{~min}$ using a spectrophotometer. The ABTS scavenging capacity of the extract was compared with that of Trolox and the activity was calculated as $\mathrm{IC}_{50}$ Trolox equivalent. All determinations were performed in triplicate. The antioxidant capacity of methanolic solutions was estimated according to the procedure described by Benzie and Strain with some modifications. Briefly, $900 \mu \mathrm{L}$ of FRAP (ferric reducing antioxidant power) reagent, prepared freshly and warmed at $37^{\circ} \mathrm{C}$, was mixed with $90 \mu \mathrm{L}$ of distilled water and $30 \mu \mathrm{L}$ of test sample. The final dilution of the test sample in the reaction mixture was $1 / 34$. The FRAP reagent contained $2.5 \mathrm{~mL}$ of a $10 \mathrm{mmol} / \mathrm{L} \mathrm{TPTZ}$ solution in $40 \mathrm{mmol} / \mathrm{L} \mathrm{HCl}$ plus $2.5 \mathrm{~mL}$ of $20 \mathrm{mmol} / \mathrm{L} \mathrm{FeCl}_{3}, 6 \mathrm{H}_{2} \mathrm{O}$ and $25 \mathrm{~mL}$ of $0.3 \mathrm{~mol} / \mathrm{L}$ acetate buffer, $\mathrm{pH}$ 3.6. The absorbance was measured at $593 \mathrm{~nm}$ against the blank after $4 \mathrm{~min}$. Methanolic solutions of known $\mathrm{Fe}(\mathrm{II})$ concentrations in the range of $100-2000 \mu \mathrm{mol} / \mathrm{L}\left(\mathrm{FeSO}_{4}, 7 \mathrm{H}_{2} \mathrm{O}\right)$ were used for calibration. FRAP value was calculated and expressed as $\mathrm{mM} \mathrm{Fe}^{2+}$ equivalent (FE) per $100 \mathrm{~g}$ sample using the calibration curve of $\mathrm{Fe}^{2+}$. All determinations were performed in triplicate. In this assay, antioxidant capacity was determined by measuring the inhibition of the volatile organic compounds and the conjugated diene hydroperoxides arising from linoleic acid oxidation. A stock solution of $\beta$-carotene/linoleic acid mixture was prepared as follows: $0.5 \mathrm{mg} \beta$-carotene $(0.9 \mathrm{mM})$ was dissolved in $1 \mathrm{~mL}$ of chloroform, then $25 \mu \mathrm{L}$ linoleic acid and $200 \mathrm{mg}$ Tween 40 was added. Then, $100 \mathrm{~mL}$ distilled water saturated with oxygen $(30 \mathrm{~min}, 100 \mathrm{~mL} / \mathrm{min}$ ) was added with vigorous shaking; $2.5 \mathrm{~mL}$ of this reaction mixture was dispensed into test tubes and $100 \mu \mathrm{L}$ portions of the methanol extracts were added; the emulsion system was incubated for up to $24 \mathrm{~h}$ at room temperature under agitation. The same procedure was repeated with the synthetic antioxidant butylated hydroxytoluene (BHT) as positive control, and a blank. After this incubation period, absorbances of the mixtures were measured at $490 \mathrm{~nm}$. The activity was calculated as \% Antioxidant Activity (AA) using the following equation: \%AA $=100 \times[1-(\mathrm{As} 0-\mathrm{Ast}) /(\mathrm{Ac0}-\mathrm{Act})]$. As 0 is the absorbance of sample at $0 \mathrm{~min}$, Ast is the absorbance of sample at $4 \mathrm{~h}, \mathrm{Ac} 0$ is the absorbance of control sample at $0 \mathrm{~min}$, and Act is the absorbance of control sample at $4 \mathrm{~h}$. All tests were run in triplicate and averaged. 


\subsection{HPLC-DAD-MS Determination of Phenolic Compounds}

B. pilosa methanol extracts were analyzed for phenol quantitative determination using a reversed-phase high performance liquid chromatography coupled to diode array and mass spectrometer (HPLC-DAD-MS) in gradient elution mode. The separation was conducted within $30 \mathrm{~min}$ of the chromatographic run, starting from the following separation conditions: $0.23 \%$ formic acid, $93 \%$ water, $7 \%$ methanol, as previously described [25]. The separation was performed on InfinityLab Poroshell 120 reverse phase column $\left(C_{18}\right.$, $150 \times 4.6 \mathrm{~mm}$ i.d., $2.7 \mu \mathrm{m}$ ) (Agilent Santa Clara, CA, USA). Column temperature was set at $30{ }^{\circ} \mathrm{C}$. Quantitative determination of phenolic compounds was performed via DAD detector. The extract was also qualitatively analyzed with an MS detector in negative ion mode, with the sole exception of rutin that was analyzed in positive ion mode. MS signal identification was realized through comparison with standard solutions and MS spectra present in the MassBank Europe database. Quantification was done through 7point calibration curves, with linearity coefficients $\left(R^{2}\right)>0.999$, in the concentration range $2-140 \mu \mathrm{g} / \mathrm{mL}$. The limits of detection were lower than $1 \mu \mathrm{g} / \mathrm{mL}$ for all assayed analytes. The area under the curve from HPLC chromatograms was used to quantify the analyte concentrations in the extract.

\subsection{Antimicrobial Tests}

In vitro antimicrobial activity of n-hexan, ethyl acetate and methanol extracts from B. pilosa were assessed against: eight bacterial strains (CLSI M07-A9), namely E. coli (ATCC 10536), E. coli PeruMycA2, E. coli PeruMycA3, B. cereus (ATCC 12826), P. aeruginosa (ATCC 15442), B. subtilis, S. typhi (clinical isolate), and S. aureus (ATCC 6538); eight dermatophytes such as T. interdigitale CCF 4823, T. tonsurans CCF 4834, T. rubrum CCF 4879, T. rubrum CCF 4933, T. rubrum CCF 4879, T. erinacei CCF 5930, A. crocatum CCF 5300, A. quadrifidum CCF 5792, Nannizzia gypsea (A. gypseum) CCF 1229; and four yeasts, namely C. tropicalis (YEPGA 6184), C. albicans (YEPGA 6379), C. parapsilosis (YEPG 6551) and C. albicans (YEPG 6138). The MICs of the plant extracts were determined in sterile 48-well microplates using the broth microdilution method of the Clinical and Laboratory Standards Institute, M07-A10 (CLSI 2015) [26]. MICs have been determined using concentrations of the dry extracts in the range 1-0.031 $\mathrm{mg} \mathrm{mL}^{-1}$, derived from serial two-fold dilutions in Mueller-Hinton Broth (MHB). For the preparation of bacterial suspensions (inocula), three to five colonies of the bacterial strains used for the test were picked from $24 \mathrm{~h}$ cultures on tryptic soy agar plates (TSA) and pre-grown overnight in Mueller-Hinton broth (MHB) to reach a cell density of approximately $1-2 \times 10^{8} \mathrm{CFU} \mathrm{mL} \mathrm{m}^{-1}$ (analogous to the $0.5 \mathrm{McF}$ arland standard). Hence, bacterial suspensions were diluted in fresh MHB and added to the MIC dilution series to reach $5 \times 10^{5} \mathrm{CFU} \mathrm{mL} \mathrm{mL}^{-1}$ in each tube. This was confirmed by plating serial dilutions of the inoculum suspensions on Mueller-Hinton Agar (MHA). The set-up included bacterial growth controls in wells containing $10 \mu \mathrm{L}$ of the test inoculum and negative controls without bacterial inoculum. MIC end-points were determined after 18-20 h incubation in ambient air at $35^{\circ} \mathrm{C}$ [27]. MIC end-points were defined as the lowest concentration of either B. pilosa extracts or ciprofloxacin that totally inhibited bacterial growth [27]. Each test was done in triplicate. Geometric means and MIC ranges were calculated. Susceptibility testing against yeasts and filamentous fungi was performed according to the CLSI M38 (CLSI 2018) and M38-Ed3 (CLSI 2017) protocols [27-29]. RPMI (Roswell Park Memorial Institute) 1640 medium (Sigma) with L-glutamine and without sodium bicarbonate, supplemented with $2 \%$ glucose $(w / v)$, buffered with $0.165 \mathrm{~mol} \mathrm{~L}^{-1}$ morpholinepropanesulphonic acid (MOPS), $\mathrm{pH} 7.0$, was used throughout the study.

The inoculum suspensions were prepared from 7-day-old cultures grown on Sabouraud Dextrose Agar (SDA; Difco) at $25^{\circ} \mathrm{C}$ and adjusted spectrophotometrically to optical densities that ranged from 0.09 to 0.11 (Mac Farland standard). Filamentous fungi (microconidia) and yeast inoculum suspensions were diluted to a ratio of 1:50 in RPMI 1640 to obtain twice an inoculum size ranging from 0.2 to $0.4 \times 10^{4-5} \mathrm{CFU} \mathrm{mL} \mathrm{m}^{-1}$. This was further confirmed by plating serial dilutions of the inoculum suspensions on SDA. MIC end-points ( $\mu \mathrm{g} \mathrm{mL}^{-1}$ ) 
were determined after $24 \mathrm{~h}$ (for yeasts) and $72 \mathrm{~h}$ (for dermatophytes) of incubation in ambient air at $30^{\circ} \mathrm{C}$ (CLSI 2017, CLSI 2018). For the plant extracts, the MIC end-points were defined as the lowest concentration that showed total growth inhibition [30]. The MIC end-points for fluconazole were defined as the lowest concentration that inhibited $50 \%$ of the growth when compared with the growth control [28]. Geometric means and MIC ranges were determined from the three biological replicates to allow comparisons between the activities of plant extracts.

\subsection{Bioinformatics}

Docking calculations were conducted through the Autodock Vina of PyRx 0.8 software, as recently described [31]. Crystal structures of target protein were derived from the Protein Data Bank (PDB) with PDB ID as follows: 5TZ1 (lanosterol 14 $\alpha$-demethylase). Discovery studio 2020 visualizer was employed to investigate the protein-ligand nonbonding interactions.

\section{Results}

\subsection{Untargeted LC-MS/MS-Based Metabolomics}

Using normalized peak intensity and principal component analysis (PCA) clustering of all annotated compounds, clear differences were observed in the metabolite profiles of the four plant parts (Figures 1 and 2; Tables 1 and 2). Although PCA analysis indicated that the different plant materials display similar metabolite profiles, oligosaccharides, disaccharides and fatty acids were found to be much more abundant in root than in the other plant parts. Monoglyceride fraction was particularly present in stem rather than in the other plant parts. By contrast, peptides and diterpenoids were found at higher levels in leaf and root, respectively, whereas amino acids showed very different distribution patterns in the four plant parts. In future experiments, to improve understanding of metabolic pathways and considering the complexity of compounds measured and their various physical and chemical properties, an internal standard for each ontology group could be used to address the differences between the extraction and ionization processes.

Table 1. B. pilosa metabolites identified by untargeted HPLC-MS analysis.

\begin{tabular}{|c|c|c|c|c|c|c|c|c|c|c|c|c|}
\hline Sample & S-r1 & S-r2 & S-r3 & R-r1 & R-r2 & R-r3 & L-r1 & L-r2 & L-r3 & SRL-r1 & SRL-r2 & SRL-r3 \\
\hline Label & Stem & Stem & Stem & Root & Root & Root & Leaf & Leaf & Leaf & $\begin{array}{l}\text { Whole } \\
\text { Plant }\end{array}$ & $\begin{array}{l}\text { Whole } \\
\text { Plant }\end{array}$ & $\begin{array}{l}\text { Whole } \\
\text { Plant }\end{array}$ \\
\hline $\begin{array}{c}\text { (10E,15Z)-9,12,13- } \\
\text { Trihydroxyoctadeca-10,15- } \\
\text { dienoic acid }\end{array}$ & 102,173 & 96,783 & 102,660 & 107,873 & 71,433 & 99,821 & 196,627 & 188,188 & 189,856 & 321,988 & 331,819 & 318,475 \\
\hline $\begin{array}{l}\text { (5.alpha.)-Androstane- } \\
\text { 3,11,17-trione }\end{array}$ & 34,194 & 36,441 & 44,320 & 0 & 8046 & 21,702 & 213,508 & 389,303 & 663,509 & 242,485 & 211,420 & 272,362 \\
\hline $\begin{array}{l}\text { (9Z,12Z)-15-Hydroxyoctadeca-9,12- } \\
\text { dienoic acid }\end{array}$ & 1065,623 & 105,5848 & 998,072 & $1,701,017$ & $1,663,171$ & $1,620,107$ & 511,408 & 411,803 & 781,119 & 772,966 & 691,404 & 827,335 \\
\hline $\begin{array}{c}\text { 1-Amino-1- } \\
\text { cyclobutanecarboxylic acid }\end{array}$ & 2669,482 & $2,636,512$ & $2,660,328$ & 143,652 & 137,926 & 133,015 & $5,333,338$ & $5,134,590$ & $5,057,910$ & $1,530,695$ & $1,489,768$ & $1,507,354$ \\
\hline 1-Kestose & 38,408 & 36,278 & 38,266 & 405,333 & 395,093 & 385,401 & 2708 & 2676 & 3990 & 95,238 & 96,636 & 93,696 \\
\hline 1-Monolinolenin & 117,654 & 76,238 & 115,568 & 9897 & 37,743 & 42,994 & 359,371 & 313,651 & 367,023 & 181,659 & 181,033 & 124,774 \\
\hline 1-Monolinoleoyl-rac-glycerol & 272,465 & 212,759 & 229,670 & 20,577 & 76,455 & 187,741 & 91,642 & 76,617 & 145,129 & 62,188 & 69,493 & 58,052 \\
\hline 1-Palmitoylglycerol & 1181,775 & $1,196,756$ & $1,197,374$ & 793,127 & 958,065 & $1,029,851$ & 938,824 & $1,007,960$ & 980,759 & 882,266 & 887,517 & 894,507 \\
\hline $\begin{array}{c}\text { 1-Propanone,1-[4-(5'-chloro-3,5- } \\
\text { dimethyl } \\
\text { [2,4'-bipyridin]-2'-yl)-1- } \\
\text { piperazinyl]-3-(methylsulfonyl)- }\end{array}$ & 27,306 & 25,892 & 27,243 & 18,541 & 9878 & 18,272 & 113,474 & 115,616 & 117,522 & 131,924 & 121,542 & 121,718 \\
\hline $\begin{array}{l}\text { 1-Stearoyl-2-hydroxy-sn-glycero-3- } \\
\text { phosphate }\end{array}$ & 97,333 & 93,382 & 83,501 & 104,747 & 108,420 & 105,850 & 98,269 & 97,664 & 105,060 & 99,102 & 95,465 & 98,623 \\
\hline $\begin{array}{l}\text { 1,4-Dibutylbenzene- } \\
\text { 1,4-dicarboxylate }\end{array}$ & 416,022 & 371,401 & 360,682 & 434,349 & 440,723 & 423,912 & 375,740 & 326,158 & 374,673 & 416,988 & 433,662 & 403,848 \\
\hline $\begin{array}{c}\text { 13-Keto-9Z,11E- } \\
\text { octadecadienoic acid }\end{array}$ & 849,783 & 806,623 & 815,040 & $1,095,797$ & $1,059,482$ & $1,052,804$ & 290,096 & 265,444 & 273,350 & 423,990 & 445,814 & 390,688 \\
\hline $\begin{array}{l}\text { 13S-Hydroxy-9Z,11E,15Z- } \\
\text { octadecatrienoic acid }\end{array}$ & 552,453 & 537,008 & 496,079 & 325,373 & 308,474 & 310,760 & $1,425,663$ & $1,557,364$ & $1,508,194$ & 933,262 & 825,946 & 908,150 \\
\hline 15-Ketofluprostenol isopropyl ester & 84,501 & 89,978 & 82,093 & 69,068 & 65,897 & 63,703 & 94,096 & 109,892 & 101,781 & 98,409 & 105,042 & 104,047 \\
\hline
\end{tabular}


Table 1. Cont.

\begin{tabular}{|c|c|c|c|c|c|c|c|c|c|c|c|c|}
\hline Sample & S-r1 & S-r2 & S-r3 & R-r1 & R-r2 & R-r3 & L-r1 & L-r2 & L-r3 & SRL-r1 & SRL-r2 & SRL-r3 \\
\hline 15-Oxo-11Z,13E-eicosadienoic acid & 25,400 & 30,232 & 15,037 & 65,219 & 376,202 & 368,532 & 37,077 & 23,581 & 35,402 & 40,086 & 30,585 & 18,520 \\
\hline $\begin{array}{c}\text { 2-[5-[(3Z,6Z)-2,12- } \\
\text { Dihydroxydodeca-3,6- } \\
\text { dienyl]oxolan-2-yl]acetic acid }\end{array}$ & 68,976 & 68,620 & 56,467 & 81,527 & 49,314 & 45,317 & 150,965 & 134,456 & 132,756 & 97,041 & 95,508 & 89,950 \\
\hline 2-Amino-2-methylpentanoic acid & $1,520,393$ & $1,553,978$ & $1,496,055$ & 827,158 & 798,054 & 810,260 & $2,691,101$ & $2,655,615$ & $2,582,067$ & $1,721,792$ & $1,718,619$ & $1,695,509$ \\
\hline 2-Chlorobenzhydrol & 47,514 & 57,401 & 55,174 & 232,441 & 90,092 & 86,206 & 21,397 & 22,254 & 21,276 & 36,777 & 33,981 & 37,482 \\
\hline 2-Palmitoyl-rac-glycerol & 987,286 & $1,009,506$ & 985,447 & 728,865 & 862,561 & 876,106 & 834,020 & 857,752 & 840,223 & 732,102 & 749,222 & 776,668 \\
\hline $\begin{array}{l}\text { 2,3-Dihydroxypropyl } \\
\text { octadecanoate }\end{array}$ & $1,133,703$ & $1,196,710$ & $1,244,961$ & $1,030,349$ & $1,164,072$ & 835,097 & $1,154,587$ & 868,611 & $1,207,623$ & 843,702 & 579,951 & 779,486 \\
\hline $\begin{array}{l}\text { 3-[2-(1,3-Benzodioxol-5-yl)-2- } \\
\text { oxoethyl]-5-bromo-3-hydroxy-1,3- } \\
\text { dihydro-2H-indol-2-one }\end{array}$ & 227,015 & 215,612 & 203,206 & 252,015 & 239,255 & 225,506 & 221,613 & 209,372 & 200,299 & 250,098 & 254,710 & 238,036 \\
\hline $\begin{array}{l}\text { 3-Cyclopentene-1-octanoic acid, } \\
\text { 2-(3-hydroxy-1-penten-1-yl)-5-oxo- }\end{array}$ & 100,681 & 107,678 & 93,923 & 62,210 & 85,529 & 82,842 & 240,640 & 231,413 & 230,760 & 209,629 & 175,091 & 174,184 \\
\hline $\begin{array}{c}\text { 3-Quinolinecarboxamide, } \\
\text { 1,4-dihydro-6-(1-methylethyl)-4- } \\
\text { oxo-1-pentyl-N- } \\
\text { tricyclo[3.3.1.1(3,7)]dec-1-yl- }\end{array}$ & 836,070 & 736,462 & 839,506 & 978,107 & 896,483 & $1,084,069$ & 843,779 & 273,146 & 671,986 & 886,706 & 548,460 & 734,240 \\
\hline $\begin{array}{l}4,4^{\prime} \text {-Dimethoxy-2'- } \\
\text { hydroxychalcone }\end{array}$ & 643,936 & 619,740 & 577,619 & 751,830 & 751,240 & 727,338 & 198,064 & 189,351 & 197,834 & 706,887 & 682,076 & 696,318 \\
\hline 4(15)-Selinene-11,12-diol & 60,495 & 62,398 & 61,881 & 6961 & 7336 & 5863 & 168,662 & 207,043 & 204,807 & 105,752 & 118,421 & 81,926 \\
\hline 5.alpha--Androstane-3,17-dione & 232,166 & 218,264 & 177,346 & $1,647,262$ & $1,626,859$ & $1,602,939$ & 271,209 & 250,080 & 75,003 & 132,367 & 126,227 & 105,284 \\
\hline 8-(3-Octyl-2-oxiranyl)octanoic acid & 343,285 & 201,933 & 158,994 & $1,477,105$ & $1,379,746$ & $1,304,038$ & 155,294 & 102,545 & 94,800 & 94,103 & 87,381 & 124,007 \\
\hline $\begin{array}{l}\text { 9-Hydroxy-9H-fluorene-9- } \\
\text { carboxylic acid } \\
\text { ethyl ester }\end{array}$ & 121,171 & 126,353 & 124,089 & 272,125 & 268,880 & 279,196 & 817 & 299 & 740 & 82,251 & 82,078 & 82,875 \\
\hline Adenosine & 124,413 & 120,181 & 125,404 & 56,006 & 59,374 & 61,803 & 171,186 & 172,298 & 175,864 & 93,050 & 87,825 & 92,119 \\
\hline $\begin{array}{l}\text { Benzyldimethyltetradecylammonium } \\
\text { cation }\end{array}$ & $3,751,915$ & $7,049,053$ & $4,888,025$ & $2 \times 10^{7}$ & $3.1 \times 10^{7}$ & $2 \times 10^{7}$ & $7,066,741$ & $7,550,522$ & $4,195,071$ & $3,096,660$ & $2,012,705$ & $5,112,836$ \\
\hline Choline cation & 42,960 & 41,793 & 28,619 & 27,102 & 26,229 & 30,563 & 184,548 & 204,572 & 206,850 & 29,087 & 31,226 & 49,225 \\
\hline cis-Vaccenic acid & 297,462 & 296,264 & 468,595 & $1,128,680$ & $1,491,160$ & $1,804,998$ & 212,669 & 448,366 & 225,255 & 100,788 & 126,485 & 163,398 \\
\hline cis,cis-9,12-Octadecadien-1-ol & $3,955,832$ & $2,099,838$ & $1,737,622$ & $1.5 \times 10^{7}$ & $8,594,468$ & $5,123,672$ & 710,151 & 474,279 & 560,286 & 667,796 & 763,629 & 368,557 \\
\hline Cynarin & 5467 & 4750 & 4372 & 124,950 & 118,904 & 129,559 & 31,993 & 31,242 & 31,062 & 39,844 & 36,948 & 40,683 \\
\hline Darendoside A & 246,997 & 243,141 & 234,194 & 156,516 & 162,866 & 154,800 & 322,945 & 334,038 & 329,086 & 301,644 & 294,323 & 305,039 \\
\hline Diisooctyl phthalate & 655,023 & 539,591 & 460,410 & 662,511 & 587,209 & 534,374 & 130,106 & 129,056 & 145,171 & 125,839 & 114,733 & 113,067 \\
\hline Gly-Gly-Val & 590,655 & 579,052 & 581,744 & 340,701 & 347,372 & 323,871 & $2,281,278$ & $2,223,467$ & $2,070,998$ & 917,095 & 867,045 & 896,749 \\
\hline L-Homoarginine & 29,419 & 8015 & 33,727 & 35,426 & 56,567 & 50,880 & 321,122 & 273,969 & 265,960 & 115,573 & 142,021 & 120,612 \\
\hline L-Tryptophan & 109,556 & 107,202 & 110,760 & 41,130 & 40,510 & 41,398 & 320,162 & 308,335 & 307,264 & 84,341 & 88,041 & 87,022 \\
\hline Labdanolic acid & 47,859 & 59,733 & 37,730 & 258,351 & 293,240 & 278,953 & 12,472 & 13,866 & 16,874 & 26,643 & 21,161 & 42,621 \\
\hline Linoleoyl ethanolamide & 151,265 & 152,477 & 146,410 & 116,457 & 116,544 & 123,219 & 32,107 & 46,621 & 33,168 & 77,323 & 94,694 & 68,613 \\
\hline Loliolide & 141,912 & 139,791 & 146,221 & 60,562 & 57,027 & 35,675 & 435,314 & 448,573 & 448,272 & 274,405 & 254,800 & 263,034 \\
\hline $\begin{array}{l}\text { Methyl arachidonyl } \\
\text { fluorophosphonate }\end{array}$ & 185,247 & 192,752 & 183,865 & 204,057 & 196,979 & 194,467 & 183,821 & 198,621 & 197,452 & 182,045 & 181,197 & 178,476 \\
\hline $\begin{array}{l}\text { N-Benzyl-N,N-dimethyl-1- } \\
\text { hexadecanaminium } \\
\text { cation }\end{array}$ & $3,372,469$ & $3,200,520$ & $1,997,818$ & $7,575,669$ & $1.3 \times 10^{7}$ & $8,243,737$ & $2,263,083$ & $1,815,563$ & $2,871,648$ & 375,748 & 582,177 & 882,638 \\
\hline N-Cyclohexyl-N'-(1-naphthyl)urea & 84,932 & 71,905 & 69,777 & 40,831 & 60,361 & 32,076 & 214,537 & 368,038 & 224,414 & 168,117 & 127,194 & 170,491 \\
\hline $\begin{array}{l}\text { N6-Carbamimidoyl-N2- } \\
\text { ((4E,6E,12E,14E)-3-hydroxy-2- } \\
\text { (hydroxymethyl)-8,10,16- } \\
\text { trimethyloctadeca-4,6,12,14- } \\
\text { tetraenoyl)lysine }\end{array}$ & 224,512 & 207,881 & 265,592 & $2,147,753$ & $2,106,907$ & $2,110,218$ & 306,836 & 266,825 & 97,710 & 189,847 & 148,730 & 175,346 \\
\hline Oleamide & 187,073 & 148,732 & 182,692 & 312,739 & 319,209 & 347,815 & 163,145 & 170,533 & 141,877 & 212,284 & 217,719 & 192,874 \\
\hline Oleoyl ethylamide & 288,452 & 278,168 & 247,724 & 381,802 & 404,893 & 409,015 & 181,339 & 206,324 & 223,119 & 866,575 & 626,771 & 825,341 \\
\hline p-Coumaric acid & 642 & 671 & 827 & 6247 & 7505 & 10,671 & 95,179 & 81,212 & 83,525 & 48,922 & 44,264 & 39,818 \\
\hline Pheophorbide a & 882,828 & 636,397 & 802,593 & 315,815 & 317,935 & 237,413 & $2,603,775$ & $4,186,462$ & $3,212,948$ & $1,264,546$ & $1,153,771$ & $1,085,994$ \\
\hline Pipericine & 140,872 & 139,382 & 135,285 & 305,766 & 368,281 & 383,514 & 291,684 & 296,772 & 169,931 & $1,184,817$ & $1,198,274$ & $1,130,158$ \\
\hline Sucrose & 171,039 & 175,897 & 181,013 & 664,452 & 656,404 & 655,640 & 30,340 & 28,754 & 32,234 & 254,888 & 246,343 & 248,963 \\
\hline Tetrahydrodicranenone B & 62,007 & 30,813 & 63,402 & 14,760 & 12,860 & 18,913 & 224,458 & 221,431 & 222,159 & 79,141 & 109,472 & 75,175 \\
\hline Timosaponin B II & 4125 & 33,780 & 20,202 & 4324 & 25,703 & 12,869 & 243,569 & 138,488 & 282,852 & 32,716 & 23,914 & 11,535 \\
\hline Tri(3-chloropropyl) phosphate & 160,488 & 159,000 & 156,995 & 329,548 & 338,360 & 288,679 & 255,460 & 253,231 & 247,410 & 340,901 & 345,598 & 352,026 \\
\hline
\end{tabular}



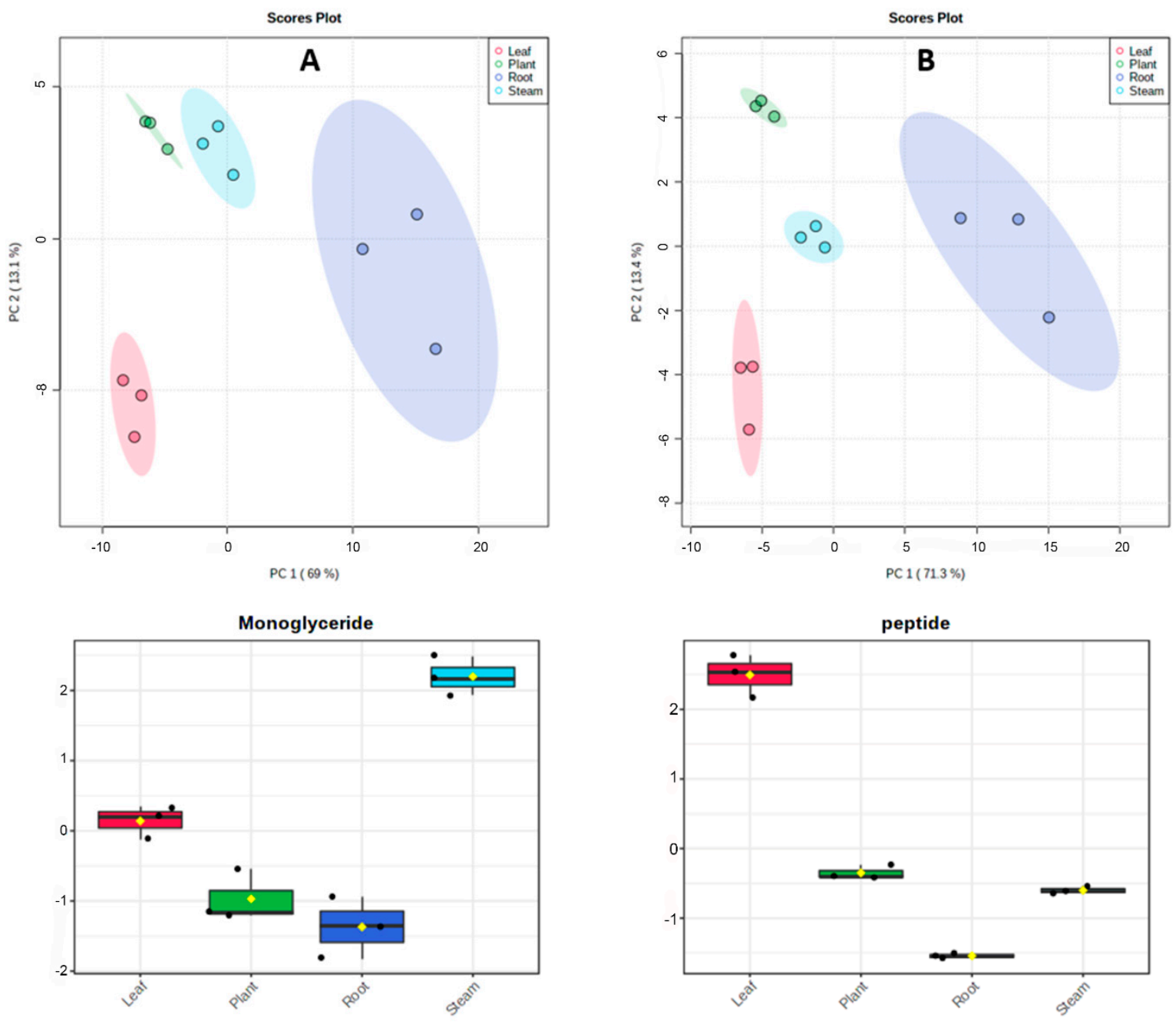

(C)

(D)
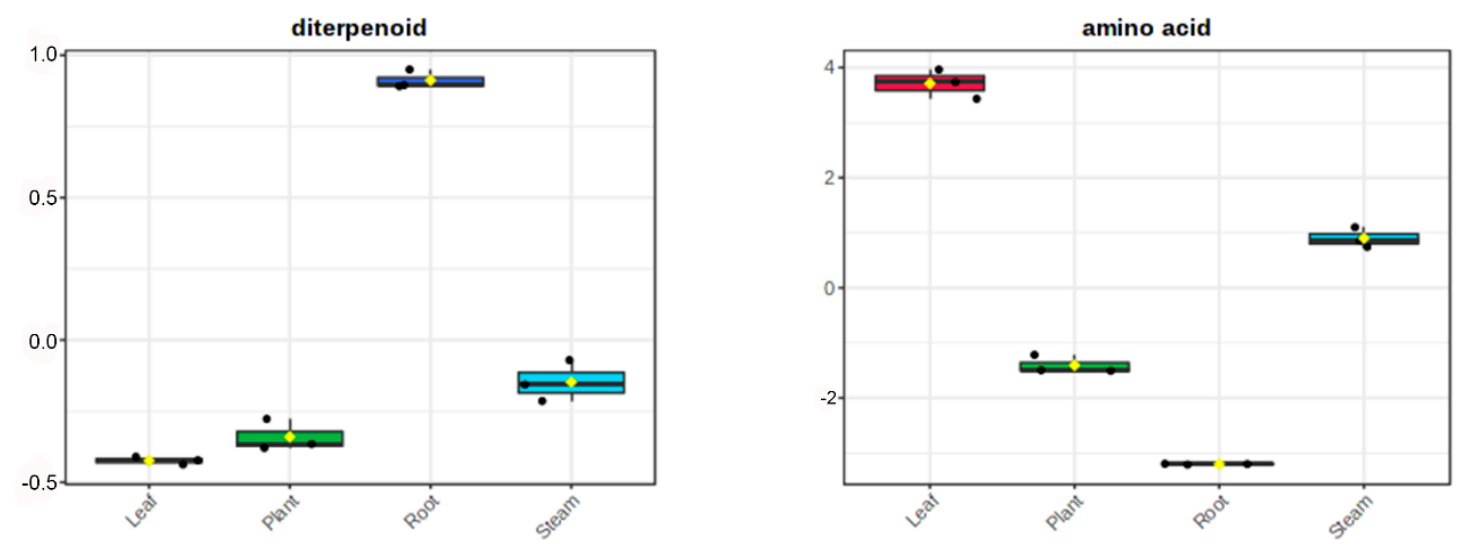

(E)

(F)

Figure 1. Principal Component Analysis (PCA) score plot of the four tested plant parts. (A) 67 annotated metabolites. (B) Ontology group of 34 annotated metabolites. Total quantitative variances of metabolites or ontology grouped metabolites were clustered to reveal the difference and relative similarities of different plant materials. 95\% confidence regions (Hotelling's T2 eclipse) are displayed for each class. Monoglyceride fraction was particularly present in stem (C), rather than in the other plant parts. By contrast, peptides and diterpenoids were found at higher levels in leaf (D) and root (E), respectively, whereas amino acids were particularly present in the leaf (F). 


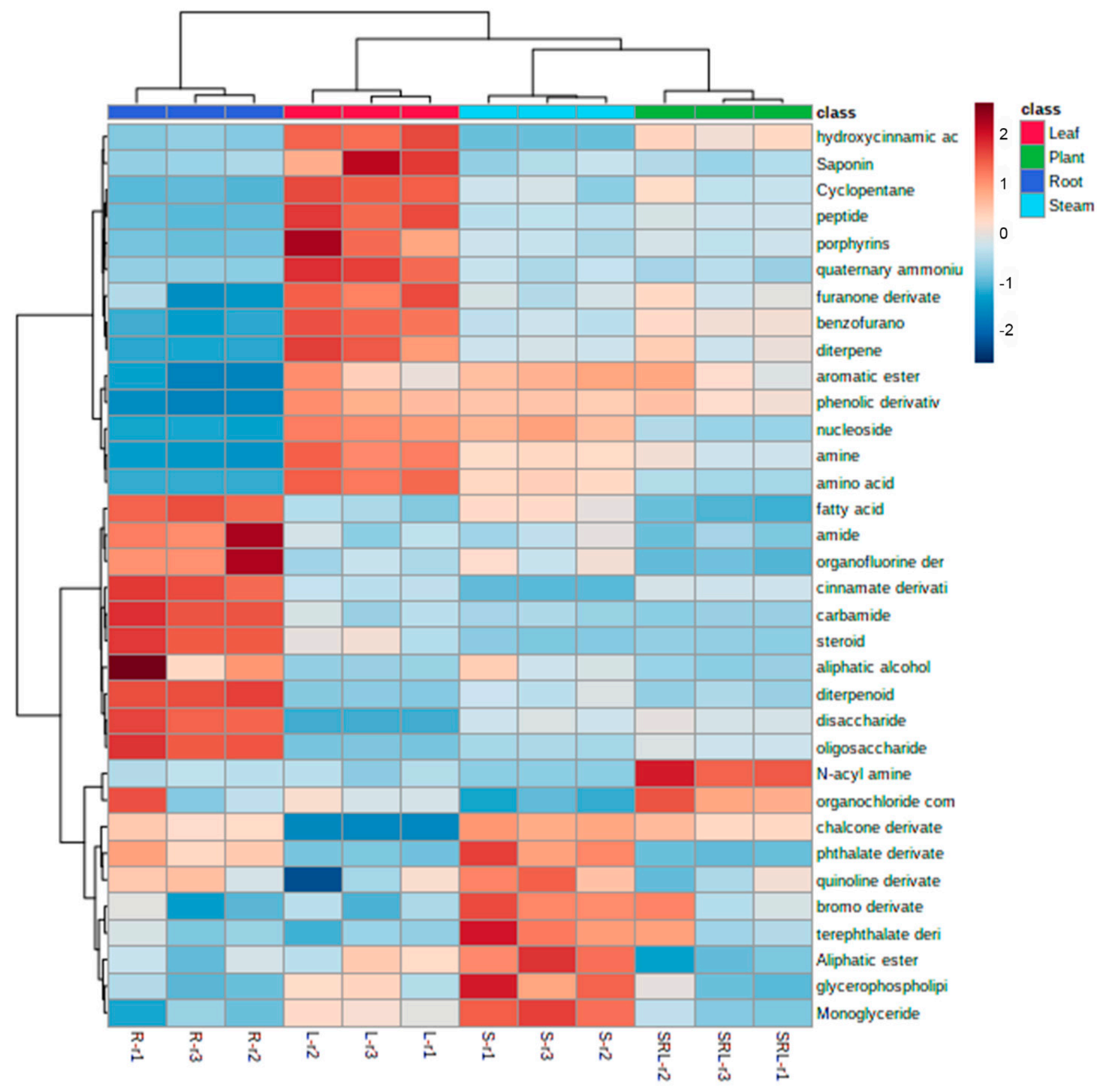

Figure 2. Cont. 


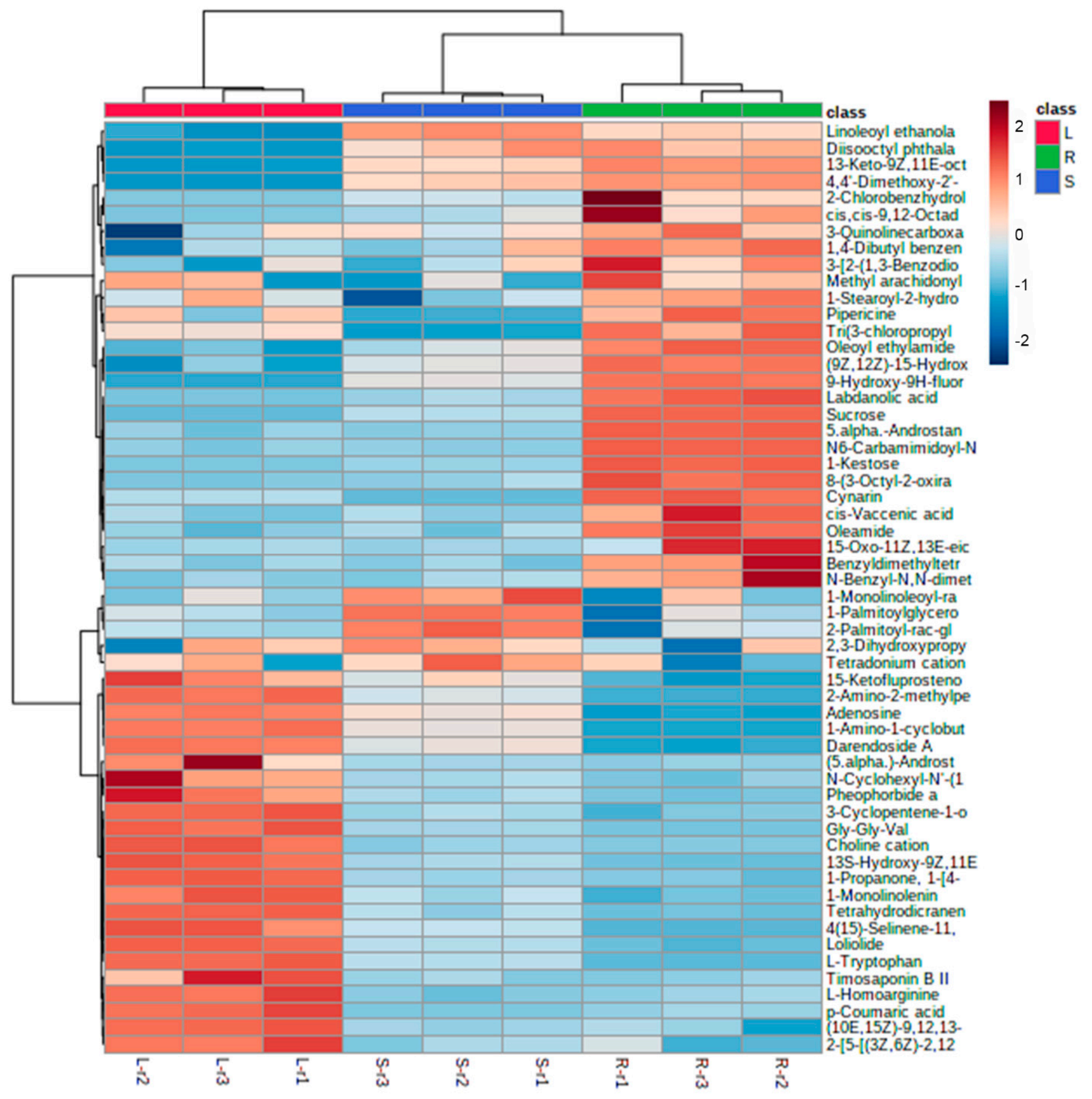

Figure 2. Heat maps showing the qualitative composition and the relative abundance of primary and secondary metabolites in root, stem, leaf and the whole plant. In the heat map columns (data reported in triplicate), red color indicates higher relative levels of metabolites, whereas the blue color suggests a minor content of them. Compound annotation was made comparing the experimental MS/MS spectra to those available in the NIST2020 Tandem Mass Spectral Library. An $m / z$ window of $0.005 \mathrm{Da}$ and a relative intensity threshold of 0.5 were selected as input parameters. Only the compounds with an identification score cut-off $>80 \%$ were retained for further analysis. Heatmap was performed with MetoboAnalyst 5.0 for either annotated metabolites or ontology grouped metabolites. Samples were normalized by median, followed by pareto scaling. 
Table 2. Ontology based-metabolomics of B. pilosa plant materials.

\begin{tabular}{|c|c|c|c|c|c|}
\hline & f.Value & $p$. Value & $\log 10(p)$ & FDR & Fisher's LSD \\
\hline amino acid & 810.05 & $2.8488 \times 10^{-10}$ & 9.5453 & $9.6858 \times 10^{-9}$ & $\begin{array}{l}\text { Leaf-Plant; Leaf-Root; Leaf-Stem; } \\
\text { Plant-Root; Stem-Plant; Stem-Root }\end{array}$ \\
\hline diterpenoid & 483.51 & $2.2226 \times 10^{-9}$ & 8.6531 & $3.3113 \times 10^{-8}$ & $\begin{array}{l}\text { Root-Leaf; Stem-Leaf; Root-Plant; } \\
\text { Stem-Plant; Root-Stem }\end{array}$ \\
\hline oligosaccharide & 420.24 & $3.8807 \times 10^{-9}$ & 8.4111 & $3.3113 \times 10^{-8}$ & $\begin{array}{l}\text { Plant-Leaf; Root-Leaf; Stem-Leaf; } \\
\text { Root-Plant; Plant-Stem; Root-Stem }\end{array}$ \\
\hline disaccharide & 419.83 & $3.8957 \times 10^{-9}$ & 8.4094 & $3.3113 \times 10^{-8}$ & $\begin{array}{c}\text { Plant-Leaf; Root-Leaf; Stem-Leaf; } \\
\text { Root-Plant; Root-Stem }\end{array}$ \\
\hline cinnamate derivative & 387.96 & $5.3299 \times 10^{-9}$ & 8.2733 & $3.6244 \times 10^{-8}$ & $\begin{array}{c}\text { Root-Leaf; Leaf-Stem; Root-Plant; } \\
\text { Plant-Stem; Root-Stem }\end{array}$ \\
\hline peptide & 339.51 & $9.0488 \times 10^{-9}$ & 8.0434 & $4.4266 \times 10^{-8}$ & $\begin{array}{c}\text { Leaf-Plant; Leaf-Root; Leaf-Stem; } \\
\text { Plant-Root; Stem-Root }\end{array}$ \\
\hline benzofurano & 333.66 & $9.6956 \times 10^{-9}$ & 8.0134 & $4.4266 \times 10^{-8}$ & $\begin{array}{l}\text { Leaf-Plant; Leaf-Root; Leaf-Stem; } \\
\text { Plant-Root; Plant-Stem; Stem-Root }\end{array}$ \\
\hline hydroxycinnamic acid & 327.68 & $1.0416 \times 10^{-8}$ & 7.9823 & $4.4266 \times 10^{-8}$ & $\begin{array}{c}\text { Leaf-Plant; Leaf-Root; Leaf-Stem; } \\
\text { Plant-Root; Plant-Stem }\end{array}$ \\
\hline nucleoside & 283.3 & $1.8539 \times 10^{-8}$ & 7.7319 & $7.0037 \times 10^{-8}$ & $\begin{array}{l}\text { Leaf-Plant; Leaf-Root; Leaf-Stem; } \\
\text { Plant-Root; Stem-Plant; Stem-Root }\end{array}$ \\
\hline chalcone derivate & 208.61 & $6.2137 \times 10^{-8}$ & 7.2067 & $2008 \times 10^{-4}$ & $\begin{array}{c}\text { Plant-Leaf; Root-Leaf; Stem-Leaf; } \\
\text { Stem-Plant; Stem-Root }\end{array}$ \\
\hline amine & 206.27 & $6.4966 \times 10^{-8}$ & 7.1873 & $2.008 \times 10^{-4}$ & $\begin{array}{l}\text { Leaf-Plant; Leaf-Root; Leaf-Stem; } \\
\text { Plant-Root; Stem-Plant; Stem-Root }\end{array}$ \\
\hline fatty acid & 176.56 & $1.199 \times 10^{-4}$ & 6.9212 & $3.3971 \times 10^{-7}$ & $\begin{array}{l}\text { Leaf-Plant; Root-Leaf; Stem-Leaf; } \\
\text { Root-Plant; Stem-Plant; Root-Stem }\end{array}$ \\
\hline quaternary ammonium compound & 165.01 & $1.5641 \times 10^{-7}$ & 6.8057 & $4.0907 \times 10^{-7}$ & $\begin{array}{c}\text { Leaf-Plant; Leaf-Root; Leaf-Stem; } \\
\text { Stem-Root }\end{array}$ \\
\hline carbamide & 149.31 & $2.3163 \times 10^{-7}$ & 6.6352 & $5.6252 \times 10^{-7}$ & Root-Leaf; Root-Plant; Root-Stem \\
\hline steroid & 128.67 & $4.1484 \times 10^{-7}$ & 6.3821 & $9.4031 \times 10^{-7}$ & $\begin{array}{c}\text { Leaf-Plant; Root-Leaf; Leaf-Stem; } \\
\text { Root-Plant; Root-Stem }\end{array}$ \\
\hline phenolic derivative & 122.17 & $5.0797 \times 10^{-7}$ & 6.2942 & $1.0794 \times 10^{-6}$ & $\begin{array}{c}\text { Leaf-Plant; Leaf-Root; Plant-Root; } \\
\text { Stem-Root }\end{array}$ \\
\hline N-acyl amine & 117.35 & $5.9443 \times 10^{-7}$ & 6.2259 & $1.1889 \times 10^{-6}$ & Plant-Leaf; Plant-Root; Plant-Stem \\
\hline Cyclopentane & 72.681 & $3.806 \times 10^{-3}$ & 5.4195 & $7.189 \times 10^{-3}$ & $\begin{array}{c}\text { Leaf-Plant; Leaf-Root; Leaf-Stem; } \\
\text { Plant-Root; Stem-Root }\end{array}$ \\
\hline Monoglyceride & 65.417 & $5.7002 \times 10^{-6}$ & 5.2441 & $1.02 \times 10^{-5}$ & $\begin{array}{c}\text { Leaf-Plant; Leaf-Root; Stem-Leaf; } \\
\text { Stem-Plant; Stem-Root }\end{array}$ \\
\hline phthalate derivate & 55.477 & $1.0687 \times 10^{-5}$ & 4.9711 & $1.8168 \times 10^{-5}$ & $\begin{array}{c}\text { Root-Leaf; Stem-Leaf; Root-Plant; } \\
\text { Stem-Plant; Stem-Root }\end{array}$ \\
\hline diterpene & 53.245 & $1.2488 \times 10^{-5}$ & 4.9035 & $2.0219 \times 10^{-5}$ & $\begin{array}{l}\text { Leaf-Plant; Leaf-Root; Leaf-Stem; } \\
\text { Plant-Root; Stem-Root }\end{array}$ \\
\hline furanone derivate & 27.794 & 0.00013939 & 3.8558 & 0.00021543 & $\begin{array}{l}\text { Leaf-Plant; Leaf-Root; Leaf-Stem; } \\
\text { Plant-Root; Stem-Root }\end{array}$ \\
\hline $\begin{array}{l}\text { Saponin } \\
\text { porphyrins }\end{array}$ & $\begin{array}{l}23.322 \\
23.314\end{array}$ & $\begin{array}{l}0.00026134 \\
0.00026165\end{array}$ & $\begin{array}{l}3.5828 \\
3.5823\end{array}$ & $\begin{array}{l}0.00036454 \\
0.00036454\end{array}$ & $\begin{array}{l}\text { Leaf-Plant; Leaf-Root; Leaf-Stem } \\
\text { Leaf-Plant; Leaf-Root; Leaf-Stem }\end{array}$ \\
\hline Aliphatic ester & 23.156 & 0.00026805 & 3.5718 & 0.00036454 & $\begin{array}{c}\text { Leaf-Plant; Stem-Leaf; Stem-Plant; } \\
\text { Stem-Root }\end{array}$ \\
\hline aromatic ester & 21.937 & 0.00032451 & 3.4888 & 0.00042436 & Leaf-Root; Plant-Root; Stem-Root \\
\hline organofluorine derivate & 21.655 & 0.00033966 & 3.469 & 0.00042771 & $\begin{array}{c}\text { Root-Leaf; Root-Plant; Stem-Plant; } \\
\text { Root-Stem }\end{array}$ \\
\hline amide & 20.619 & 0.00040342 & 3.3942 & 0.00048987 & Root-Leaf; Root-Plant; Root-Stem \\
\hline glycerophospholipid & 14.443 & 0.0013585 & 2.867 & 0.0015927 & Stem-Leaf; Stem-Plant; Stem-Root \\
\hline terephthalate derivate & 9.818 & 0.0046632 & 2.3313 & 0.0052849 & Stem-Leaf; Stem-Plant; Stem-Root \\
\hline bromo derivate & 8.1485 & 0.008147 & 2.089 & 0.0089355 & Stem-Leaf; Stem-Root \\
\hline aliphatic alcohol & 6.3184 & 0.016665 & 1.7782 & 0.017707 & Root-Leaf; Root-Plant; Root-Stem \\
\hline organochloride compound & 5.4374 & 0.024748 & 1.6065 & 0.025498 & Plant-Stem; Root-Stem \\
\hline
\end{tabular}

\subsection{Antimicrobial Effects}

The antimicrobial effects of n-hexane, ethyl acetate and methanol extracts were compared with reference drugs and presented in Tables 3-5. Overall, clinical Gram-negative bacterial strains (PeruMyc 2, 3, 5 and 7) showed a somewhat lower susceptibility to plant extracts than that of Gram-positive ones. This was particularly true for the B. cereus strain PeruMycA 4, that showed the lowest MIC values (Table 3). Regardless of the bacterial strain used, n-hexane extracts showed the lowest antibacterial activity (Table 3). Table 4 shows the MIC ranges and geometric means of plant extract and fluconazole against the yeast species tested. C. parapsilosis (YEPGA 6551) were the most sensitive yeast strain to plant extracts, with MIC ranges of $<0.031-0.198 \mathrm{mg} \mathrm{mL}^{-1}$, while C. albicans (YEPGA 6379) showed the least sensitivity to the plant extract. 
Table 3. Minimal inhibitory concentrations (MICs) of B. pilosa n-hexane, ethyl acetate and methanol extracts and ciprofloxacin against clinical Gram-positive and Gram-negative bacteria.

\begin{tabular}{|c|c|c|c|c|c|}
\hline & & \multicolumn{4}{|c|}{ Minimum Inhibitory Concentration (MIC) } \\
\hline \multicolumn{2}{|c|}{ Extract Typology } & n-hex (mg mL $\left.L^{-1}\right)$ & EtOAc $\left(\mathrm{mg} \mathrm{mL}^{-1}\right)$ & $\mathrm{MeOH}\left(\mathrm{mg} \mathrm{mL}^{-1}\right)$ & Ciprofloxacin \\
\hline Bacterial strain & Plant parts & & & & \\
\hline \multirow[t]{4}{*}{ E. coli (ATCC 10536) } & roots & $<0.031$ & $<0.031$ & $<0.031$ & $<0.12$ \\
\hline & leaves & $<0.031$ & $<0.031$ & $<0.031$ & \\
\hline & stems & $<0.031$ & $<0.031$ & $<0.031$ & \\
\hline & whole & $<0.031$ & $<0.031$ & $<0.031$ & \\
\hline \multirow{4}{*}{ E. coli (PeruMycA 2) } & roots & $0.049(0.031-0.062)$ & $0.078(0.062-0.125)$ & $0.157(0.125-0.25)$ & $1.23(0.98-1.95)$ \\
\hline & leaves & $0.049(0.031-0.062)$ & $0.039(0.031-0.062)$ & $0.157(0.125-0.25)$ & \\
\hline & stems & $0.039(0.031-0.62)$ & $0.157(0.125-0.25)$ & $0.157(0.125-0.25)$ & \\
\hline & whole & $<0.031$ & $0.157(0.125-0.25)$ & $0.157(0.125-0.25)$ & \\
\hline \multirow{4}{*}{ E. coli (PeruMycA 3) } & roots & $0.099(0.062-0.125)$ & $0.157(0.125-0.25)$ & $0.157(0.125-0.25)$ & $0.62(0.49-0.98)$ \\
\hline & leaves & $0.198(0.125-0.25)$ & $0.039(0.031-0.062)$ & $0.198(0.125-0.25)$ & \\
\hline & stems & $0.078(0.062-0.125)$ & $0.315(0.25-0.5)$ & $0.157(0.125-0.25)$ & \\
\hline & whole & $0.039(0.031-0.62)$ & $0.157(0.125-0.25)$ & $0.157(0.125-0.25)$ & \\
\hline \multirow[t]{4}{*}{ B. cereus (PeruMycA 4) } & roots & $<0.031$ & $<0.031$ & $<0.031$ & $<0.12$ \\
\hline & leaves & $<0.031$ & $<0.031$ & $<0.031$ & \\
\hline & stems & $<0.031$ & $<0.031$ & $<0.031$ & \\
\hline & whole & $<0.031$ & $<0.031$ & $<0.031$ & \\
\hline \multirow[t]{4}{*}{ P. aeruginosa (ATCC15442) } & roots & $0.039(0.031-0.062)$ & $0.078(0.062-0.125)$ & $<0.031$ & $<0.12$ \\
\hline & leaves & $0.049(0.031-0.062)$ & $0.039(0.031-0.062)$ & $<0.031$ & \\
\hline & stems & $0.039(0.031-0.062)$ & $0.039(0.031-0.062)$ & $<0.031$ & \\
\hline & whole & $0.039(0.031-0.62)$ & $0.078(0.062-0.125)$ & $<0.031$ & \\
\hline \multirow[t]{4}{*}{ B. subtilis (PeruMyc 6) } & roots & $0.315(0.5-0.25)$ & $0.198(0.125-0.25)$ & $0.078(0.062-0.125)$ & $<0.12$ \\
\hline & leaves & $0.396(0.25-0.5)$ & $0.396(0.25-05)$ & $0.078(0.062-0.125)$ & \\
\hline & stems & $0.315(0.5-0.25)$ & $0.198(0.125-0.25)$ & $<0.031$ & \\
\hline & whole & $0.315(0.25-0.5)$ & $0.098(0.062-0.125)$ & $<0.031$ & \\
\hline \multirow[t]{4}{*}{ S. typhy (PeruMyc 7) } & roots & $0.049(0.031-0.062)$ & $0.157(0.125-0.25)$ & $0.157(0.125 .0 .25)$ & 0.49 \\
\hline & leaves & $0.198(0.125-0.25)$ & $0.198(0.125-0.25)$ & $0.078(0.062-0.125)$ & \\
\hline & stems & $0.198(0.125-0.25)$ & $0.039(0.031-0.062)$ & $0.049(0.031-0.062)$ & \\
\hline & whole & $0.157(0.125-0.25)$ & $0.198(0.125-0.25)$ & $0.049(0.031-0.062)$ & \\
\hline \multirow[t]{4}{*}{ S. aureus (ATCC 6538) } & roots & $0.039(0.031-0.062)$ & $0.039(0.031-0.062)$ & $0.039(0.031-0.062)$ & 0.98 \\
\hline & leaves & $0.049(0.031-0.062)$ & $0.039(0.031-0.062)$ & $0.039(0.031-0.062)$ & \\
\hline & stems & $0.039(0.031-0.062)$ & $0.039(0.031-0.062)$ & $0.039(0.031-0.062)$ & \\
\hline & whole & $0.039(0.031-0.062)$ & $0.039(0.031-0.062)$ & $0.039(0.031-0.062)$ & \\
\hline
\end{tabular}

Table 4. Minimal inhibitory concentrations (MICs) of B. pilosa n-hexane, ethyl acetate and methanol extracts and fluconazole against clinical yeasts.

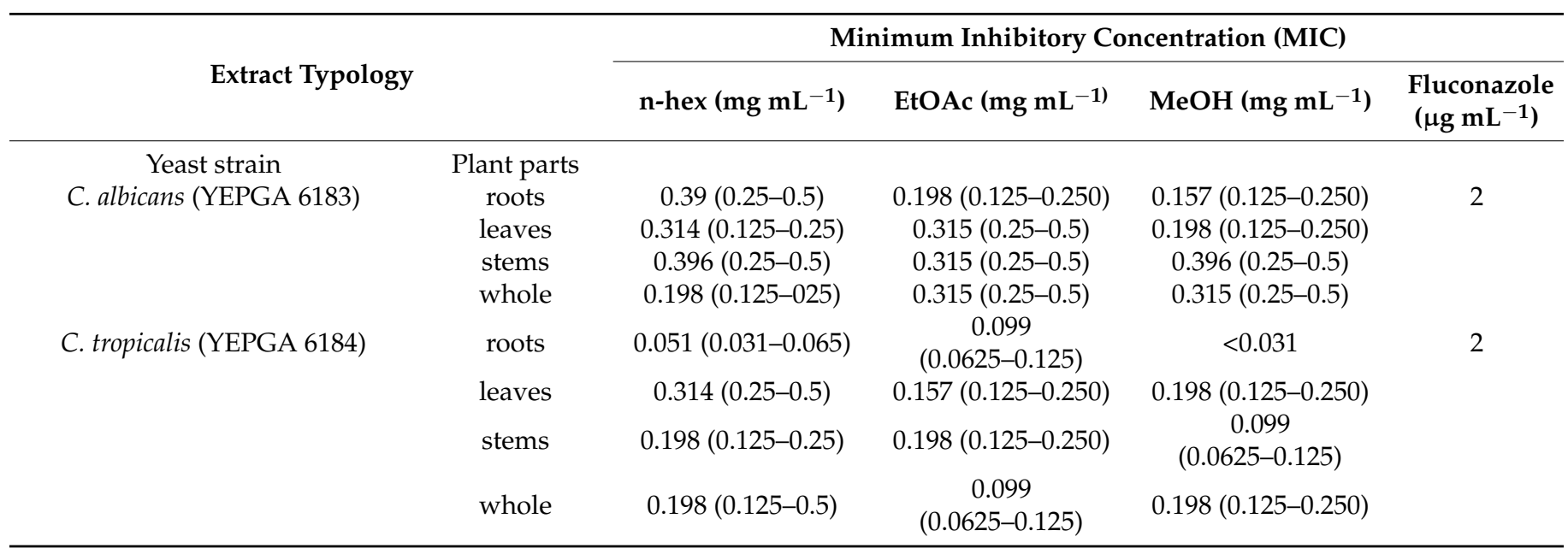


Table 4. Cont.

\begin{tabular}{|c|c|c|c|c|c|}
\hline \multirow[b]{2}{*}{ Extract Typology } & & \multicolumn{4}{|c|}{ Minimum Inhibitory Concentration (MIC) } \\
\hline & & n-hex $\left(\mathrm{mg} \mathrm{mL}^{-1}\right)$ & EtOAc $\left(\mathrm{mg} \mathrm{mL}^{-1}\right)$ & $\mathrm{MeOH}\left(\mathrm{mg} \mathrm{mL}^{-1}\right)$ & 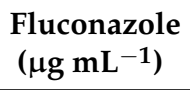 \\
\hline C. albicans (YEPGA 6379) & roots & $0.314(0.25-0.5)$ & $\begin{array}{c}0.099 \\
(0.0625-0.125)\end{array}$ & $0.157(0.125-0.250)$ & 1 \\
\hline & $\begin{array}{l}\text { leaves } \\
\text { stems } \\
\text { whole }\end{array}$ & $\begin{array}{c}0.396(0.25-0.5) \\
0.314(0.250-0.5) \\
0.314(0.125-0.5)\end{array}$ & $\begin{array}{c}0.198(0.125-0.250) \\
0.315(0.25-0.5) \\
0.315(0.25-0.5)\end{array}$ & $\begin{array}{c}0.198(0.125-0.250) \\
0.396(0.25-0.5) \\
0.315(0.25-0.5)\end{array}$ & \\
\hline C. parapsilosis (YEPGA 6551) & $\begin{array}{l}\text { roots } \\
\text { leaves } \\
\text { stems } \\
\text { whole }\end{array}$ & $\begin{array}{c}<0.031 \\
0.157(0.125-0.25) \\
0.198(0.125-025) \\
<0.031\end{array}$ & $\begin{array}{l}<0.031 \\
<0.031 \\
<0.031 \\
<0.031\end{array}$ & $\begin{array}{l}<0.031 \\
<0.031 \\
<0.031 \\
<0.031\end{array}$ & 4 \\
\hline
\end{tabular}

Table 5. Minimal inhibitory concentrations (MICs) of B. pilosa n-hexane, ethyl acetate, methanol extracts and griseofulvin against clinical dermatophytes.

\begin{tabular}{|c|c|c|c|c|c|}
\hline \multirow{2}{*}{\multicolumn{2}{|c|}{ Extract Typology }} & \multicolumn{4}{|c|}{ Minimum Inhibitory Concentration (MIC) } \\
\hline & & \multirow[t]{2}{*}{$\mathrm{n}-\mathrm{h} \times\left(\mathrm{mg} \mathrm{mL} \mathrm{L}^{-1}\right)$} & \multirow[t]{2}{*}{ EtOAc $\left(\mathrm{mg} \mathrm{mL}^{-1}\right)$} & \multirow[t]{2}{*}{$\mathrm{MeOH}\left(\mu \mathrm{g} \mathrm{mL}{ }^{-1}\right)$} & \multirow[t]{2}{*}{$\begin{array}{l}\text { Griseofulvin }(\mu \mathrm{g} \\
\left.\mathrm{mL}^{-1}\right)\end{array}$} \\
\hline Fungal strain & Plant parts & & & & \\
\hline \multirow{4}{*}{$\begin{array}{l}\text { T. rubrum } \\
\text { (CCF4933) }\end{array}$} & roots & $0.198(0.125-0.25)$ & $<0.031$ & $<0.031$ & $1.26(1-2)$ \\
\hline & leaves & $0.396(0.25-0.5)$ & $<0.031$ & $0.049(0.031-0.062)$ & \\
\hline & stems & $0.315(0.25-0.5)$ & $0.049(0.031-0.062)$ & $0.157(0.125-0.25)$ & \\
\hline & whole & $0.396(0.25-0.5)$ & $<0.031$ & $<0.031$ & \\
\hline \multirow{4}{*}{$\begin{array}{l}\text { T. mentagrofites } \\
\text { (CCF 4823) }\end{array}$} & roots & $0.198(0.125-0.25)$ & $<0.031$ & $<0.031$ & 1 \\
\hline & leaves & $0.049(0.031-0.062)$ & $<0.031$ & $<0.031$ & \\
\hline & stems & $0.315(0.25-0.5)$ & $0.049(0.031-0.062)$ & $0.157(0.125-0.25)$ & \\
\hline & whole & $0.396(0.25-0.5)$ & $<0.031$ & $<0.031$ & \\
\hline \multirow{4}{*}{$\begin{array}{l}\text { T.rubrum } \\
\text { (CCF4879) }\end{array}$} & roots & $0.198(0.125-0.25)$ & $<0.031$ & $<0.031$ & 2 \\
\hline & leaves & $0.198(0.125-2.5$ & $<0.031$ & $0.049(0.031-0.062)$ & \\
\hline & stems & $0.315(0.25-0.5)$ & $<0.031$ & $0.157(0.125-0.25)$ & \\
\hline & whole & $0.396(0.25-0.5)$ & $<0.031$ & $<0.031$ & \\
\hline \multirow{4}{*}{$\begin{array}{l}\text { T. tonsurans } \\
\text { (CCF4834) }\end{array}$} & roots & $0.198(0.125-0.25)$ & $<0.031$ & $<0.031$ & 0.125 \\
\hline & leaves & $0.049(0.031-0.062)$ & $<0.031$ & $198(0.125-25))$ & \\
\hline & stems & $0.315(0.25-0.5)$ & $<0.031$ & $0.157(0.125-0.25)$ & \\
\hline & whole & $0.396(0.25-0.5)$ & $<0.031$ & $<0.031$ & \\
\hline \multirow{4}{*}{$\begin{array}{l}\text { A. crocatum } \\
\text { (CCF5300) }\end{array}$} & roots & $0.198(0.125-0.25)$ & $<0.031$ & $<0.031$ & $>8$ \\
\hline & leaves & $0.396(0.25-0.5)$ & $<0.031$ & $0.078(0.062-0.125)$ & \\
\hline & stems & $0.315(0.25-0.5)$ & $0.049(0.031-0.062)$ & $0.157(0.125-0.25)$ & \\
\hline & whole & $0.396(0.25-0.5)$ & $<0.031$ & $<0.031$ & \\
\hline \multirow{4}{*}{$\begin{array}{l}\text { A. gypseum } \\
\text { (CCF6261) }\end{array}$} & roots & $0.315(0.25-0.5)$ & $<0.031$ & $<0.031$ & $1.587(1-2)$ \\
\hline & leaves & $0.396(0.25-0.5)$ & $<0.031$ & $0.157(0.125-0.25)$ & \\
\hline & stems & $0.198(0.125-0.25)$ & $0.315(0.25-0.5)$ & $0.078(0.062-0.125)$ & \\
\hline & whole & $0.198(0.125-0.25)$ & $<0.031$ & $<0.031$ & \\
\hline \multirow{4}{*}{$\begin{array}{l}\text { T. erinacei } \\
\text { (CCF5930) }\end{array}$} & roots & $0.315(0.25-0.5)$ & $<0.031$ & $<0.031$ & 0.25 \\
\hline & leaves & $0.396(0.25-0.5)$ & $<0.031$ & $0.157(0.125-0.25)$ & \\
\hline & stems & $0.198(0.125-0.25)$ & $0.157(0.125-0.250)$ & $0.157(0.125-0.25)$ & \\
\hline & whole & $0.198(0.125-0.25)$ & $<0.031$ & $<0.031$ & \\
\hline \multirow{4}{*}{$\begin{array}{l}\text { A. quadrifidum } \\
\text { (CCF5792) }\end{array}$} & roots & $0.315(0.25-0.5)$ & $<0.031$ & $<0.031$ & $>8$ \\
\hline & leaves & $0.396(0.25-0.5)$ & $<0.031$ & $0.157(0.125-0.25)$ & \\
\hline & stems & $0.198(0.125-0.25)$ & $0.157(0.125-0.250)$ & $0.157(0.125-0.25)$ & \\
\hline & whole & $0.396(0.25-0.5)$ & $<0.031$ & $<0.031$ & \\
\hline
\end{tabular}




\subsection{Phenolic Profile}

The HPLC analyses showed that gallic acid, caftaric acid, catechin, chlorogenic acid, epicatechin and caffeic acid were present in most of the analyzed plant materials (Figure 3A-D), with the only exception of leaf methanol extract. Among the identified compounds, caftaric acid (peak \#3 in the Figure 3A-C; retention time: $9.15 \mathrm{~min}$.) was revealed to be the prominent phenolic compound, especially in the extract prepared from the whole plant $(3.03 \mu \mathrm{g} / \mathrm{mL})$.

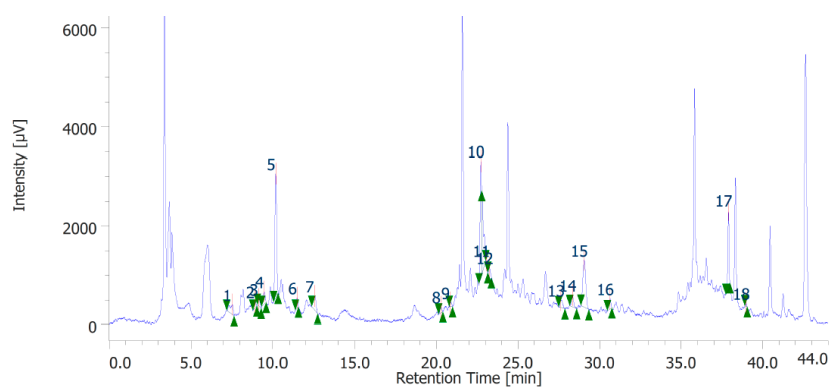

(A) Whole plant
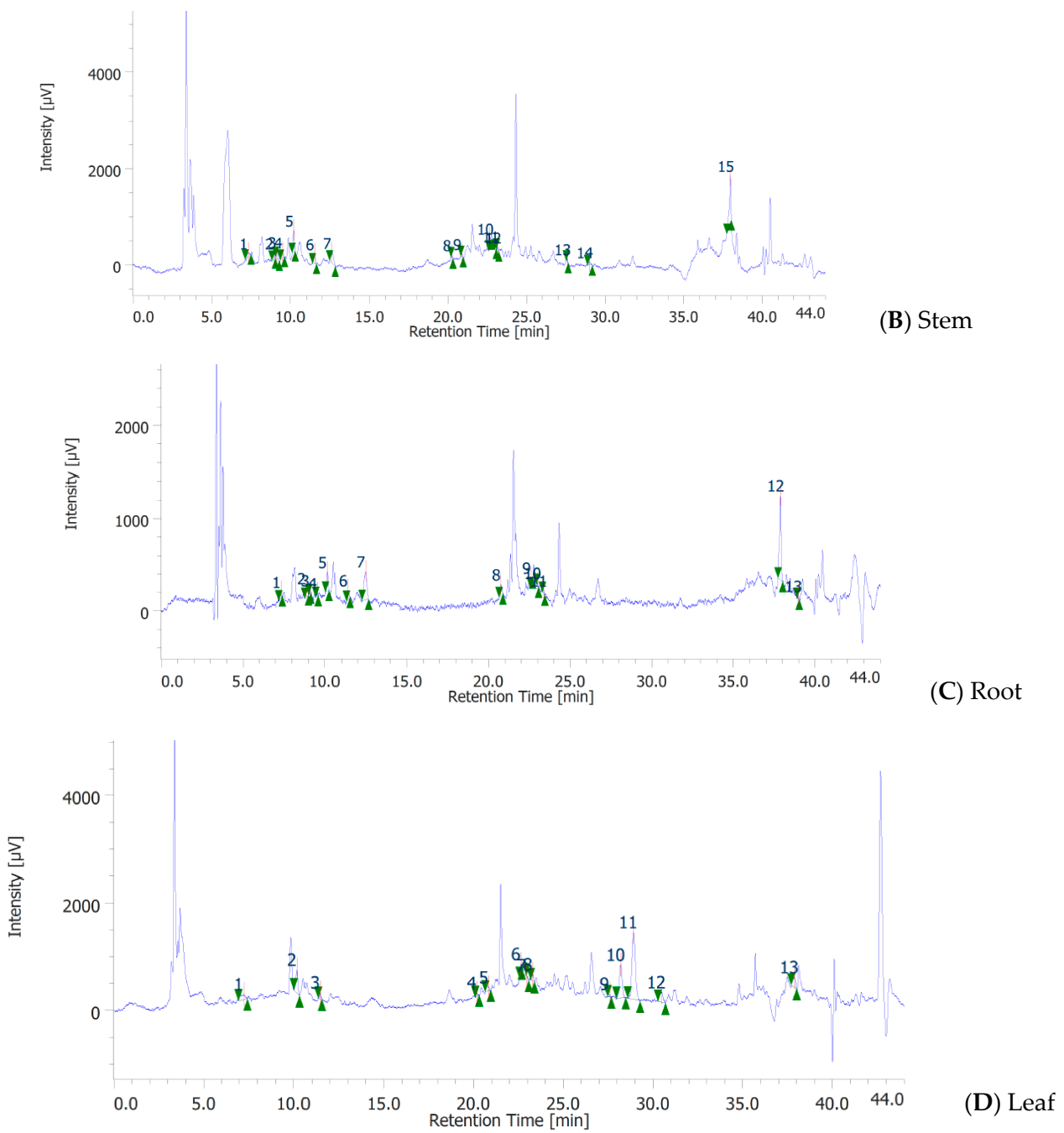

Figure 3. HPLC-DAD-MS analysis of methanolic extracts from B. pilosa plant materials. Among identified phenolic compounds in Table 1, caftaric acid (peak \#3), catechin (peak \#4), chlorogenic acid (peak \#5) and epicatechin (peak \#6) were identified in the whole plant (A), stem (B) and root (C). In the leaf (D), the principal phenolics identified in the extract were: gallic acid (peak\#1), chlorogenic acid (peak\#2) and epicatechin (peak \#3). 


\subsection{In Silico Experiments}

The results of the in silico experiment highlight the capability of caftaric acid to interact with the active site of the enzyme with a micromolar affinity, through the formation of both hydrogen bonds and alkyl interactions (Figure 4). The affinity of the caftaric acid was compared to that of the reference drug ketoconazole that, as expected, shows a much higher (sub-micromolar) affinity compared to that of caftaric acid.
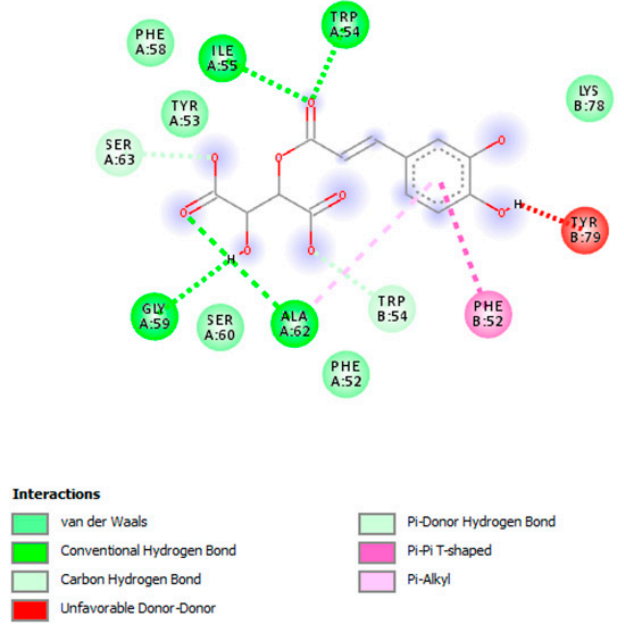

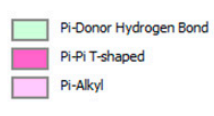

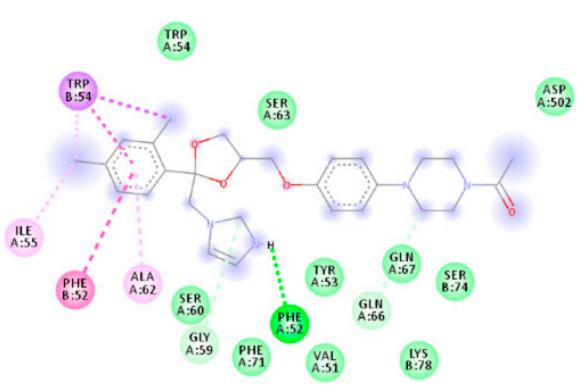

A

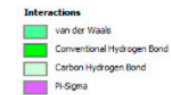

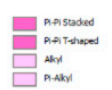

B

Figure 4. (A) Putative interactions between caftaric acid and lanosterol 14 $\alpha$-demethylase (PDB ID: 5TZ1). Free energy binding and affinity are $-6.8 \mathrm{Kcal} / \mathrm{mol}$ and $10.5 \mu \mathrm{M}$, respectively. (B) Putative interactions between ketoconazole with lanosterol 14 $\alpha$-demethylase (PDB ID: 5TZ1). Free energy binding and affinity are $-9.6 \mathrm{Kcal} / \mathrm{mol}$ and $0.1 \mu \mathrm{M}$, respectively.

\subsection{Intrinsic Scavenging/Reducing Properties}

Finally, the radical scavenging/reducing properties of the methanol extracts of B. pilosa plant materials were assayed (Table 6). The values of the DPPH assay are reported in comparison with the activity of the Trolox and the best activity is shown by the leaves with a rather good mean value of $9.9 \mathrm{IC}_{50}$ referred to the Trolox. Decidedly low is the activity of the stems with a mean value of 101.4, whilst the roots have a medium-low mean value (15.2). The values of the ABTS assay are reported in comparison with the activity of the Trolox and analogously to the DPPH test the best activity is shown by the leaves, with a rather good mean value of $15.4 \mathrm{IC}_{50}$ referred to the Trolox. Decidedly low is the activity of the stems with a mean value of 89.1, whilst the roots have a medium-low mean value (25.3). The FRAP assay shows the mM Fe(II)+ equivalent (FE) for an $100 \mathrm{~g}$ sample; interesting is the activity of the leaves, with a mean value of 73.2, while much lower are the values of the other extracts: plants $>$ roots $>$ stems with values of 17.7-15.3-10.1, respectively. In Beta Carotene/Linoleic acid assay values are expressed as \% of antioxidant activity. The extracts do not show pro-oxidant activity but a good antioxidant value of the leaves (44.7) and of the plant (37.4). The antioxidant action of the stems is less than that of the leaves in all the tests carried out and the methanolic extract follows the order leaves $>$ plants $>$ roots $>$ stems.

Table 6. Scavenging/reducing properties of methanol extracts from roots, stems leaves and whole plants of B. pilosa.

\begin{tabular}{ccccc}
\hline Plant Material & DPPH & ABTS & FRAP & $\begin{array}{c}\beta- \\
\text { Carotene/Linoleic } \\
\text { Acid Assay }\end{array}$ \\
\hline roots & $15.2 \pm 1.22$ & $25.3 \pm 2.16$ & $15.3 \pm 1.17$ & $21.1 \pm 1.74$ \\
Stems & $101.4 \pm 8.23$ & $89.1 \pm 7.81$ & $10.1 \pm 0.87$ & $44.7 \pm 3.86$ \\
leaves & $9.9 \pm 0.84$ & $15.4 \pm 1.36$ & $73.2 \pm 6.5$ & $27.4 \pm 2.32$ \\
plants & $3.7 \pm 2.38$ & $34.3 \pm 3.05$ & $17.7 \pm 1.49$ & $37.4 \pm 3.78$ \\
\hline
\end{tabular}




\section{Discussion}

Considering the traditional ethnopharmacology and phytotherapy uses [6,7], in the present study, different materials from B. pilosa have been assayed in order to unravel plant material composition and extracts' antimicrobial effects. Oligosaccharide, disaccharide and fatty acids were found to be much more abundant in root than in the other plant parts. Monoglycerides were more abundant in stem than in the other plant parts, whereas peptide and diterpenoid were more abundant in leaf and root, respectively. By contrast, amino acids showed very different distribution patterns in the four plant parts. The microbiological study investigated the potential anti-bacterial and anti-fungal effects of the extracts against selected pathogen strains. All tested extracts showed fungal growth inhibition; particularly active were the ethyl acetate and methanolic extracts from root and leaves that showed the highest antifungal activity among all samples tested. Ethyl acetate and methanolic extract were also classified as potent for dermatophyte when compared to n-hexane. The results clearly demonstrated that the extracts were less effective when compared to the reference drugs, namely the anti-bacterial ciprofloxacin and the anti-mycotic fluconazole and griseofulvin. Nevertheless, the ethyl acetate and methanol extracts of $B$. pilosa displayed anti-mycotic activity on C. albicans (YEPGA 6379) and dermatophytes; this deserves further investigation. Considering the results of the antimicrobial tests pointing to promising activity of polar extracts from B. pilosa as anti-bacterial and anti-mycotic agents, a quali-quantitative HPLC-DAD-MS analysis was conducted on phenolic acids and flavonoids from B. pilosa methanol extracts, in order to unravel the putative mechanisms underlying the observed antimicrobial effects. In this regard, it is sensitive to highlight that phenolic compounds could explain, albeit partially, the anti-mycotic effects induced by polar extracts [32,33]. Specifically, the HPLC analyses showed that gallic acid, caftaric acid, catechin, chlorogenic acid, epicatechin and caffeic acid were present in most of the analyzed plant materials. Caftaric acid is known to be a phytocompound characterizing Echinacea species [34]. However, different studies suggest the presence of this phenolic compound in Bidens species, including B. tripartita and B. pilosa $[28,29,35,36]$, thus further corroborating the results of the present phytochemical investigation. A docking approach was also conducted in order to predict putative interactions between the caftaric acid and the lanosterol $14 \alpha$-demethylase, playing a master role in fungal metabolism. The results of the in silico experiment highlight the capability of caftaric acid to interact with the active site of the enzyme with a micromolar affinity, through the formation of both hydrogen bonds and alkyl interactions. The putative affinity of caftaric acid towards the selected target enzyme was lower compared to that of the reference drug ketoconazole. However, this putative affinity is consistent with the concentration of the phenolic compound in the extract, and also with the extract MIC values, above all against the Candida species. Therefore, the present docking experiments highlight the importance of phenolic compounds in mediating, albeit partially, the antimicrobial effects induced by B. pilosa methanolic extracts. Finally, the radical scavenging/reducing properties of the methanol extracts of B. pilosa plant materials were assayed. The intrinsic antioxidant effects of the extracts were evaluated through ABTS, DPPH and Beta-Carotene assays. The antioxidant action of the stems is less than that of the leaves in all of the tests carried out and the methanolic extract follows the order leaves $>$ plants $>$ roots $>$ stems. Antioxidants attract a growing interest owing to their protective roles against oxidative deterioration in food and in the body, and against oxidative stress-mediated pathological processes. Screening of antioxidant properties of plants requires appropriate methods, which address the mechanism of antioxidant activity and focus on the kinetics of the reactions including the antioxidants. Many studies evaluating the antioxidant activity of various samples of research interest using different methods in food and human health have been conducted. Methods based on inhibited autoxidation are the most suited for termination-enhancing antioxidants and for chain-breaking antioxidants. In general, the methods for the determination of the antioxidant capacity of plant extract can deactivate radicals by two major mechanisms and were divided into two major groups: assays based on the single electron transfer (SET) 
reaction, and assays based on hydrogen atom transfer (HAT). The end result is the same, regardless of mechanism, but kinetics and potential for side reactions are different. SET-based methods detect the ability of a potential antioxidant to transfer one electron to reduce any compound, including metals, carbonyls, and radicals [37]. HAT-based methods measure the ability of an antioxidant to quench free radicals by hydrogen donation [38]. For this purpose, the most common methods used in vitro determination of antioxidant capacity of plant raw extract were considered in this manuscript. The methanol extracts of roots, stems, leaves, and whole plants were tested with DPPH assay (1,1-diphenyl-2-picrylhydrazyl radical scavenging), ABTS assay (2,2'-azinobis-(3-ethylbenzothiazoline-6-sulphonate) radical scavenging), FRAP assay (ferric reducing antioxidant power), and beta carotene/linoleic acid assay (double bond antioxidant power). Methods based on the HAT reaction include the $\beta$-Carotene bleaching assays [37]. The SET-based methods include the following assays: 2,2-Diphenyl-1-picrylhydrazyl radical scavenging assay (DPPH·), ferric ion reducing antioxidant power assay (FRAP), and 2,2-Azinobis 3-ethylbenzthiazoline-6-sulfonic acid radical scavenging assay (ABTS). It was reported that ABTS methods used both HAT and SET mechanisms [38]. Phenolic compounds are secondary plant metabolites naturally present in almost all plant materials, including food products of plant origin. Many of the health-protective effects of phenolic compounds have been ascribed to their antioxidant, anticarcinogenic, antimutagenic, antimicrobial, anti-inflammatory and other biological properties $[39,40]$. The correlation matrix for Pearson coefficients provides a high correlation with the FRAP assay (0.90) and a very low inverse correlation with the linoleic assay $(-0.53)$; intermediate values were for DPPH $(-0.70)$ and ABTS $(-0.74)$ assays. Flavonoids are cyclized diphenylpropanes that commonly occur in plants and particularly plant foods. They are polyphenolic compounds, which are very effective antioxidants that serve against chronic diseases. The intrinsic antioxidant properties have also been related to enzyme inhibition properties [41]. Flavonoids have been isolated from almost all parts of the plant such as leaves, stems, roots, fruits, or seeds. In general, the effective antioxidant ability of flavonoids depends on some factors: the metal-chelating potential that is strongly dependent on the arrangement of hydroxyls and carbonyl group around the molecule, the presence of hydrogen or electron-donating substituents able to reduce free radicals, and the ability of the flavonoid to delocalize the unpaired electron leading to formation of a stable phenoxy radical [39]. Similarly, the correlation matrix for Pearson coefficients provides a high correlation with the FRAP assay (0.88), a very low inverse correlation with the linoleic assay (-0.32), and intermediate values for DPPH (-0.64) and ABTS (-0.68) assays. The chemical complexity of the extracts, stemming from the fact that they are often mixtures of many compounds, with differences in functional groups, polarity, and chemical behavior, could lead to unpredictable results about their possible antioxidant activity.

\section{Conclusions}

The results of antimicrobial susceptibility testing were analyzed and thoroughly discussed, also with respect to results from similar studies. It should be born in mind, however, that comparisons between bioactivity results are always difficult, because working protocols may differ in terms of extraction methods, test organisms and test systems used [42]. In the present study, the microbiological assays pointed to the promising activity of polar extracts, namely methanol extracts, as anti-mycotic agents. The anti-mycotic effect could be partially mediated by phenolic compounds detected by colorimetric and HPLC-DAD-MS analyses. The pattern of phenolic compound composition could also explain the intrinsic scavenging/reducing properties of the methanol extracts. The present phytochemical determinations also validated previous studies suggesting the presence of caftaric acid, in the phytocomplex of B. pilosa [35]. Additionally, considering the intrinsic anti-inflammatory properties of caftaric acid [43], we cannot exclude its involvement in mediating, albeit partially, the anti-inflammatory effects of B. pilosa [44]. Therefore, future studies could be conducted in order to investigate anti-inflammatory effects induced by the present extracts from B. pilosa. 
Author Contributions: Conceptualization, P.A., G.O., L.M. and C.F.; Methodology, P.A., L.M., G.O. and C.F.; Software, C.F.; Validation, P.A., G.O., L.M. and C.F.; Formal analysis, C.F.; Investigation, G.A.F., F.M., B.T., R.V., R.M.P., C.E. and L.V.; Resources, G.O. and C.F.; Data curation, P.A., G.O. and L.M.; Writing-original draft preparation, P.A.; Writing-review and editing, C.F., L.M. and G.O.; Visualization, R.V. and B.T.; Supervision, R.V.; Project administration, P.A., C.F., L.M. and G.O.; Funding acquisition, C.F., L.M. and G.O. All authors have read and agreed to the published version of the manuscript.

Funding: The study was supported by Italian Ministry funds (FAR 2020), granted to Giustino Orlando, Luigi Menghini and Claudio Ferrante.

Institutional Review Board Statement: Not applicable.

Informed Consent Statement: Not applicable.

Data Availability Statement: Not applicable.

Acknowledgments: The present article is part of the third mission activities of the Botanic Garden "Giardino dei Semplici" planned for the 20th anniversary of the establishment.

Conflicts of Interest: The authors declare no conflict of interest.

\section{References}

1. Ferrante, C.; Angelini, P.; Venanzoni, R.; Angeles Flores, G.; Tirillini, B.; Recinella, L.; Chiavaroli, A.; Brunetti, L.; Leone, S.; Di Simone, S.C.; et al. Antimicrobial, antioxidant, and antiproliferative effects of Coronilla minima: An unexplored botanical species. Antibiotics 2020, 9, 611. [CrossRef]

2. Mahomoodally, M.F.; Jugreet, S.; Sinan, K.I.; Zengin, G.; Ak, G.; Ceylan, R.; Jek̋, J.; Cziáky, Z.; Angelini, P.; Angeles Flores, G.; et al. Pharmacological potential and chemical characterization of Bridelia ferruginea Benth. A native tropical African medicinal plant. Antibiotics 2021, 10, 223. [CrossRef]

3. Chiavaroli, C.; Sinan, K.I.; Zengin, G.; Mahomoodally, M.F.; Sadeer, N.B.; Etienne, O.K.; Cziáky, Z.; Jeko, J.; Glamocilja, J.; Sokovic, M.; et al. Identification of chemical profiles and biological properties of Rhizophora racemosa G. Mey. extracts obtained by different methods and solvents. Antioxidants 2020, 9, 533. [CrossRef] [PubMed]

4. Ferrante, C.; Chiavaroli, A.; Angelini, P.; Venanzoni, R.; Angeles Flores, G.; Brunetti, L.; Petrucci, M.; Politi, M.; Menghini, L.; Leone, S.; et al. Phenolic content and antimicrobial and anti-Inflammatory effects of Solidago virga-aurea, Phyllanthus niruri, Epilobium angustifolium, Peumus boldus, and Ononis spinosa extracts. Antibiotics 2020, 9, 783. [CrossRef] [PubMed]

5. Xuan, T.D.; Khanh, T.D. Chemistry and pharmacology of Bidens pilosa: An overview. J. Pharm. Investig. 2016, 46, 91-132. [CrossRef] [PubMed]

6. Bartolome, A.P.; Villaseñor, I.M.; Yang, W.C. Bidens pilosa L. (Asteraceae): Botanical properties, traditional uses, phytochemistry, and pharmacology. Evid. Based Complement. Alternat. Med. 2013, 2013, 340215. [CrossRef]

7. Yang, H.L.; Chen, S.C.; Chang, N.W.; Chang, J.M.; Lee, M.L.; Tsai, P.C.; Fu, H.H.; Kao, W.W.; Chiang, H.C.; Wang, H.H.; et al. Protection from oxidative damage using Bidens pilosa extracts in normal human erythrocytes. Food Chem. Toxicol. 2006, 44, 1513-1521. [CrossRef]

8. Brandão, M.G.; Krettli, A.U.; Soares, L.S.; Nery, C.G.; Marinuzzi, H.C. Antimalarial activity of extracts and fractions from Bidens pilosa and other Bidens species (Asteraceae) correlated with the presence of acetylene and flavonoid compounds. J. Ethnopharmacol. 1997, 57, 131-138. [CrossRef]

9. Kumari, P.; Misra, K.; Sisodia, B.S.; Faridi, U.; Srivastava, S.; Luqman, S.; Darokar, M.P.; Negi, A.S.; Gupta, M.M.; Singh, S.C.; et al. A promising anticancer and antimalarial component from the leaves of Bidens pilosa. Planta Med. 2009, 75, 59-61. [CrossRef]

10. Kviecinski, M.R.; Felipe, K.B.; Correia, J.F.; Ferreira, E.A.; Rossi, M.H.; de Moura Gatti, F.; Filho, D.W.; Pedrosa, R.C. Brazilian Bidens pilosa Linné yields fraction containing quercetin-derived flavonoid with free radical scavenger activity and hepatoprotective effects. Libyan J. Med. 2011, 6. [CrossRef]

11. Ubillas, R.P.; Mendez, C.D.; Jolad, S.D.; Luo, J.; King, S.R.; Carlson, T.J.; Fort, D.M. Antihyperglycemic acetylenic glucosides from Bidens pilosa. Planta Med. 2000, 66, 82-83. [CrossRef]

12. Geissberger, P.; Séquin, U. Constituents of Bidens pilosa L.: Do the components found so far explain the use of this plant in traditional medicine? Acta Trop. 1991, 48, 251-261. [CrossRef]

13. Khan, M.R.; Kihara, M.; Omoloso, A.D. Antimicrobial activity of Bidens pilosa, Bischofia javanica, Elmerillia papuana and Sigesbekia orientalis. Fitoterapia 2001, 72, 662-665. [CrossRef]

14. Motsei, M.L.; Lindsey, K.L.; Van Staden, J.; Jägerm, A.K. Screening of traditionally used South African plants for antifungal activity against Candida albicans. J. Ethnopharmacol. 2003, 86, 235-241. [CrossRef]

15. Abajo, C.; Boffill, M.A.; Campo, J.D.; Mendez, M.A.; Gonzalez, Y.; Mitjans, M.; Vinardell, M.P. In vitro study of the antioxidant and immunomodulatory activity of aqueous infusion of Bidens pilosa. J. Ethnopharmacol. 2004, 93, 319-323. [CrossRef] [PubMed] 
16. Rojas, J.J.; Ochoa, V.J.; Ocampo, S.A.; Muñoz, J.F. Screening for antimicrobial activity of ten medicinal plants used in Colombian folkloric medicine: A possible alternative in the treatment of non-nosocomial infections. BMC Complement. Altern. Med. 2006, 6, 2. [CrossRef] [PubMed]

17. Deba, F.; Xuan, T.D.; Yasuda, M.; Tawata, S. Chemical composition and antioxidant, antibacterial and antifungal activities of the essential oils from Bidens pilosa Linn. var. radiata. Food Control 2008, 19, 346-352. [CrossRef]

18. Ashafa, A.O.T.; Afolayan, A.J. Screening the root extracts from Biden pilosa L. var. radiata (Asteraceae) for antimicrobial potentials. J. Med. Plant Res. 2009, 3, 568-572.

19. Silva, J.J.; Cerdeira, C.D.; Chavasco, J.M.; Cintra, A.B.P.; Silva, C.B.P.; Mendonça, A.N.; Ishikawa, T.; Boriollo, M.F.G.; Chavasco, J.K. In vitro screening antibacterial activity of Bidens pilosa Linné and Annona crassiflora Mart. against oxacillin resistant Staphylococcus aureus (ORSA) from the aerial environment at the dental clinic. Rev. Inst. Med. Trop. Sao Paulo 2014, 56, 333-340. [CrossRef]

20. Shandukani, P.D.; Tshidino, S.C.; Masoko, P.; Moganedi, K.M. Antibacterial activity and in situ efficacy of Bidens pilosa Linn and Dichrostachys cinerea Wight et Arn extracts against common diarrhoea-causing waterborne bacteria. BMC Complement. Altern. Med. 2018, 18, 171. [CrossRef]

21. Chiang, Y.M.; Chuang, D.Y.; Wang, S.Y.; Kuo, Y.H.; Tsai, P.W.; Shyur, L.F. Metabolite profiling and chemopreventive bioactivity of plant extracts from Bidens pilosa. J. Ethnopharmacol. 2004, 95, 409-419. [CrossRef] [PubMed]

22. Muchuweti, M.; Mupure, C.; Ndhlala, A.; Murenje, T.; Benhura, M.A.N. Screening of antioxidant and radical scavenging activity of Vigna ungiculata, Bidens pilosa and Cleome gynandra. Am. J. Food Technol. 2007, 2, 161-168. [CrossRef]

23. Deba, F.; Xuan, T.D.; Yasuda, M.; Tawata, S. Herbicidal and fungicidal activities and identification of potential phytotoxins from Bidens pilosa L. var. radiata Scherff. Weed Biol. Manag. 2007, 7, 77-83. [CrossRef]

24. Nthulane, N.P.; Mosebi, S.; Tshikalange, T.E.; Nyila, M.A.; Mankga, L.T. Antimicrobial and anti-inflammatory activities of selected medicinal plants against pathogens causing sexually transmitted infections. J. Herbmed. Pharmacol. 2020, 9, 130-137. [CrossRef]

25. Di Giacomo, V.; Recinella, L.; Chiavaroli, A.; Orlando, G.; Cataldi, A.; Rapino, M.; Di Valerio, V.; Politi, M.; Antolini, M.D.; Acquaviva, A.; et al. Metabolomic profile and antioxidant/anti-Inflammatory effects of industrial hemp water extract in fibroblasts, keratinocytes and isolated mouse skin specimens. Antioxidants 2021, 10, 44. [CrossRef]

26. CLSI. Methods for Dilution Antimicrobial Susceptibility Tests for Bacteria that Grow Aerobically; Approved Standard, 10th ed.; CLSI document M07-A10; Clinical and Laboratory Standards Institute: Wayne, PA, USA, 2015.

27. Covino, S.; D’Ellena, E.; Tirillini, B.; Angeles Flores, G.; Arcangeli, A.; Bistocchi, G.; Venanzoni, R.; Angelini, P. Characterization of biological activities of methanol extract of Fuscoporia torulosa (Basidiomycetes) from Italy. Int. J. Med. Mushrooms 2019, 21, 1051-1063. [CrossRef]

28. CLSI. Reference Method for Broth Dilution Antifungal Susceptibility Testing of Filamentous Fungi, 3rd ed.; CLSI standard M38; Clinical Laboratory Standards Institute: Wayne, PA, USA, 2017.

29. CLSI. Reference Method for Broth Dilution Antifungal Susceptibility Testing of Yeasts. Approved Standard; Document M38; Clinical Laboratory Standards Institute: Wayne, PA, USA, 2018.

30. Pagiotti, R.; Angelini, P.; Rubini, A.; Tirillini, B.; Granetti, B.; Venanzoni, R. Identification and characterisation of human pathogenic filamentous fungi and susceptibility to Thymus schimperi essential oil. Mycoses 2011, 54, e364-e376. [CrossRef]

31. Angelini, P.; Venanzoni, R.; Angeles Flores, G.; Tirillini, B.; Orlando, G.; Recinella, L.; Chiavaroli, A.; Brunetti, L.; Leone, S.; Di Simone, S.C.; et al. Evaluation of antioxidant, antimicrobial and tyrosinase inhibitory activities of extracts from Tricholosporum goniospermum, an edible wild mushroom. Antibiotics 2020, 9, 513. [CrossRef]

32. Ferrante, C.; Recinella, L.; Ronci, M.; Menghini, L.; Brunetti, L.; Chiavaroli, A.; Leone, S.; Di Iorio, L.; Carradori, S.; Tirillini, B.; et al. Multiple pharmacognostic characterization on hemp commercial cultivars: Focus on inflorescence water extract activity. Food Chem. Toxicol. 2019, 125, 452-461. [CrossRef]

33. Bottari, N.B.; Lopes, L.Q.; Pizzuti, K.; Filippi Dos Santos Alves, C.; Corrêa, M.S.; Bolzan, L.P.; Zago, A.; de Almeida Vaucher, R.; Boligon, A.A.; Giongo, J.L.; et al. Antimicrobial activity and phytochemical characterization of Carya illinoensis. Microb. Pathog. 2017, 104, 190-195. [CrossRef]

34. Waidyanatha, S.; Pierfelice, J.; Cristy, T.; Mutlu, E.; Burback, B.; Rider, C.V.; Ryan, K. A strategy for test article selection and phytochemical characterization of Echinacea purpurea extract for safety testing. Food Chem. Toxicol. 2020, 137, 111125. [CrossRef]

35. Ramabulana, A.-T.; Steenkamp, P.A.; Madala, N.E.; Dubery, I.A. Application of plant growth regulators modulates the profile of chlorogenic acids in cultured Bidens pilosa cells. Plants 2021, 10, 437. [CrossRef]

36. Śliwa, K.; Sikora, E.; Ogonowski, J.; Oszmiański, J.; Kolniak-Ostek, J. A micelle mediated extraction as a new method of obtaining the infusion of Bidens tripartita. Acta Biochim. Pol. 2016, 63, 543-548. [CrossRef]

37. Huang, D.; Ou, B.; Prior, R.L. The chemistry behind antioxidant capacity assays. J. Agric. Food Chem. 2005, 53, 1841-1856. [CrossRef]

38. Prior, R.L.; Wu, X.; Schaich, K. Standardized methods for the determination of antioxidant capacity and phenolics in foods and dietary supplements. J. Agric. Food Chem. 2005, 53, 4290-4302. [CrossRef]

39. Gülçin, I.; Topal, F.; Çakmakçı, R.; Bilsel, M.; Gören, A.C.; Erdogan, U. Pomological features, nutritional quality, polyphenol content analysis, and antioxidant properties of domesticated and 3 wild ecotype forms of raspberries (Rubus idaeus L.). J. Food Sci. 2011, 76, 585-593. [CrossRef] [PubMed]

40. Naczk, M.; Shahidi, F. Phenolics in cereals, fruits and vegetables: Occurrence, extraction and analysis. J. Pharm. Biomed. Anal. 2006, 41, 1523-1542, Erratum in: 2007, 43, 798. [CrossRef] 
41. Chatatikun, M.; Supjaroen, P.; Promlat, P.; Chantarangkul, C.; Waranuntakul, S.; Nawarat, J.; Tangpong, J.; Chiabchalard, A. Antioxidant and tyrosinase inhibitor properties of an aqueous extract of Garcinia atrovirdis Griff. Ex. T. Anderson fruit pericarps. Pharmacogn. J. 2020, 12, 71-78. [CrossRef]

42. Angelini, P.; Tirillini, B.; Bistocchi, G.; Arcangeli, A.; Rubini, A.; Pellegrino, R.M.; Fabiani, R.; Cruciani, G.; Venanzoni, R.; Rosignoli, P. Overview of the biological activities of a methanol extract from wild red belt conk, Fomitopsis pinicola (Agaricomycetes), fruiting bodies from Central Italy. Int. J. Med. Mushrooms 2018, 20, 1047-1063. [CrossRef]

43. Tanyeli, A.; Ekinci Akdemir, F.N.; Eraslan, E.; Güler, M.C.; Nacar, T. Anti-oxidant and anti-inflamatuar effectiveness of caftaric acid on gastric ulcer induced by indomethacin in rats. Gen. Physiol Biophys. 2019, 38, 175-181. [CrossRef] [PubMed]

44. Yoshida, N.; Kanekura, T.; Higashi, Y.; Kanzaki, T. Bidens pilosa suppresses interleukin-1beta-induced cyclooxygenase-2 expression through the inhibition of mitogen activated protein kinases phosphorylation in normal human dermal fibroblasts. J. Dermatol. 2006, 33, 676-683. [CrossRef] [PubMed] 